\title{
A study of the expanding envelope of Nova V1974 Cyg 1992 based on IUE high resolution spectroscopy ${ }^{\star}$
}

\author{
A. Cassatella ${ }^{1,2}$, H. J. G. L. M. Lamers ${ }^{3}$, C. Rossi $^{4}$, A. Altamore ${ }^{2}$, and R. González-Riestra ${ }^{5}$ \\ 1 Istituto di Astrofisica Spaziale e Fisica Cosmica, CNR, Area di Ricerca Tor Vergata, Via del Fosso del Cavaliere 100, \\ 00133 Roma, Italy \\ 2 Dipartimento di Fisica E. Amaldi, Università degli Studi Roma Tre, Via della Vasca Navale 84, 00146 Roma, Italy \\ 3 Astronomical Institute and SRON Laboratory for Space Research, Utrecht University, Princetonplein 5, 3584CC, Utrecht, \\ The Netherlands \\ ${ }^{4}$ Dipartimento di Fisica, Università degli Studi La Sapienza, Piazzale A. Moro 2, 00185 Roma, Italy \\ 5 XMM Observatory, Villafranca Satellite Tracking Station, PO Box 50727, 28080 Madrid, Spain
}

Received 23 July 2003 / Accepted 12 February 2004

\begin{abstract}
We have carried out a detailed analysis of the IUE archival high resolution spectra of the classical nova V1974 Cyg 1992. The main UV resonance lines show P Cygni profiles in the first days, which change into symmetric pure emission lines, and then slowly become fainter and narrower. Lines of higher ionization species reach their peak luminosity later than those of low ionization. This can be explained by a fast wind which is optically thick in the early days, when the pseudophotosphere is located inside the wind. As the mass loss decreases, the radius of the pseudo-photosphere schrinks. This has three effects that explain the observed changes: (1) the deeper accelerating layers of the wind become visible where the emission lines are formed by collisional excitation and/or recombination; (2) as the mass loss rate decreases the emission comes from deeper regions of the wind where the velocities are smaller; (3) the effective temperature and the degree of ionization increase. In addition to the P Cygni and emission lines, we could identify two shortward shifted absorption systems which originate in two separate expanding shells, outside the wind layers where the emission lines are formed. The velocity of both shells increase with time. The outer main shell, containing most of the matter ejected at the outburst, produces the so-called "principal absorption line system", and the inner faster moving second shell produces the so-called "diffuse-enhanced absorption line system". The acceleration of the two shells is the result of increasing line-radiation pressure due to the UV-brightening of the star as the effective radius decreases. Around day 60 the second shell has overtaken the slower moving principal system shell, and merged with it. This explains: the sudden disappearance of the diffuse line system near that date, the upward jump of $\Delta v=240 \mathrm{~km} \mathrm{~s}^{-1}$ in velocity of the principal system and the first detection of hard X-ray emission on day 63 . This velocity jump indicates that the main shell is $\approx 4$ times more massive than the second shell. The deceleration suffered by the diffuse-enhanced system after the shock provides a shock temperature $T_{\text {shock }} \approx 1.6 \mathrm{keV}$, in fairly good agreement with the temperature of the observed hard $\mathrm{X}$-ray emission. The UV observations are interpreted through an empirical model in which the pre-nova slow wind phase is followed by the ejection of two shells, where the principal and the diffuse-enhanced absorption systems are formed, and by a phase of fast continuous lower density wind. Our empirical expansion velocity law for the principal system, together with $\mathrm{H} \alpha$ interferometric observations of the angular radius on day 10 are used to determine the distance to the nova, which is found to be $2.9 \pm 0.2 \mathrm{kpc}$, in agreement with $H S T$ imaging and with the absolute magnitude versus rate of decline relationship.
\end{abstract}

Key words. stars: novae, cataclysmic variables - techniques: spectroscopic - ultraviolet: stars - X-rays: stars

\section{Introduction}

V1974 Cyg (Nova Cygni 1992) was discovered in outburst by Collins (1992) on 1992 February 19.07 UT (JD = 2448672.3 ) at a visual magnitude $V=6.8$. The discovery time will be taken as day zero all through this paper.

Send offprint requests to: A. Cassatella,

e-mail: cassatella@fis.uniroma3.it

* Tables in Appendix are only available in electronic form at the CDS via anonymous ftp to

cdsarc.u-strasbg.fr $(130.79 .128 .5)$ or via

http://cdsweb.u-strasbg.fr/cgi-bin/qcat?J/A+A/420/571
The true time of the outburst of V1974 Cyg can be roughly estimated by comparing its visual light curve, characterized by an optical decay time $t_{3}=42 \pm 2$ days (Chochol et al. 1993), with that of two novae having a very similar value of $t_{3}$ of about 40 days: N Lac 1910.9 and N Mon 1918.0. The rise time from quiescence to maximum is $\approx 8$ days for both objects (cf. the light curves in Cecchini \& Gratton 1942). We can then estimate, by analogy, that the outburst of V1974 Cyg took place about 8 days before optical maximum, i.e. about 4.6 days before the reported discovery. An independent guess of the effective date of outburst can be made by extrapolating the 
pre-maximum light curve of V1974 Cyg to the magnitude at quiescence, as quoted in Andrillat \& Houziaux (1993). We obtain in this case that the outburst took place at least 2 days before the time of discovery. By averaging the two estimates, we obtain that the outburst has probably taken place about 3.3 days before the discovery time (our adopted day zero).

V1974 Cyg has immediately caught the attention of observers because of its exceptional brightness at maximum ( $V_{\max } \approx+4.4$ visual magnitude) reached on February 22.5 (Rosino et al. 1996) and consequently, as being an easy target for multiwavelength observations, which were soon extended to the ultraviolet, radio, infrared and X-rays. These abundant data represent a unique opportunity to get insight into important questions concerning the nova phenomenon.

In this paper we study the dynamics of the expanding envelope of V1974 Cyg through the analysis of the velocity variations of the ultraviolet absorption lines. Our main purpose is to get insight on the velocity law of the absorption systems, on their origin and possible interaction, and on the velocity and ionization stratification within the envelope.

In general the optical spectra of classical novae show four systems of shortward shifted absorption lines (McLaughlin 1960). In order of appearance, of increasing radial velocity and degree of ionization these systems are: the pre-maximum, the principal, the diffuse-enhanced and the Orion systems. (For a critical review of the controversial interpretation of the absorption line systems in novae see Friedjung \& Duerbeck 1993). In this paper we will adopt this classification scheme.

\section{Observations}

The difficulty of line identifications in the ultraviolet spectra of novae during early outburst is sensibly alleviated in the case of V1974 Cygni thanks to the very large amount of spectra (about 330) obtained with the IUE satellite at both high and low resolution covering roughly 0.8 and 3.3 years of the nova evolution, respectively.

We mainly concentrate on the analysis of the high resolution observations of V1974 Cyg (about 140 spectra), which cover 200 days and 330 days of its post-outburst evolution in the SWP and LWP cameras, respectively, at a resolution of about $25 \mathrm{~km} \mathrm{~s}^{-1}$. However, there is unfortunately an observational gap between day 53 and 60, where important changes have probably taken place, as discussed later. The data were retrieved from the INES (IUE Newly Extracted Spectra) system through its Principal Centre at http: //ines. laeff.esa.es. A full description of the INES system for high resolution spectra is given in González-Riestra et al. (2000).

We have also used IUE low resolution spectra to highlight specific aspects of the flux variations which cannot be studied at high resolution given the $\approx 90$ times lower detection efficiency. In Fig. 1 we plot as a function of time the ultraviolet continuum flux in a band $20 \AA$ wide centered at $1455 \AA$, and the flux of some strong emission lines covering a wide range of ionization energy. A characteristic feature of the emission lines is that the higher their degree of ionization, the later they reach a flux maximum: the maximum emission takes place on day 21 for Mg II $2800 \AA$, day 42 for O I $1300 \AA$, day 77 for C III] $1909 \AA$,

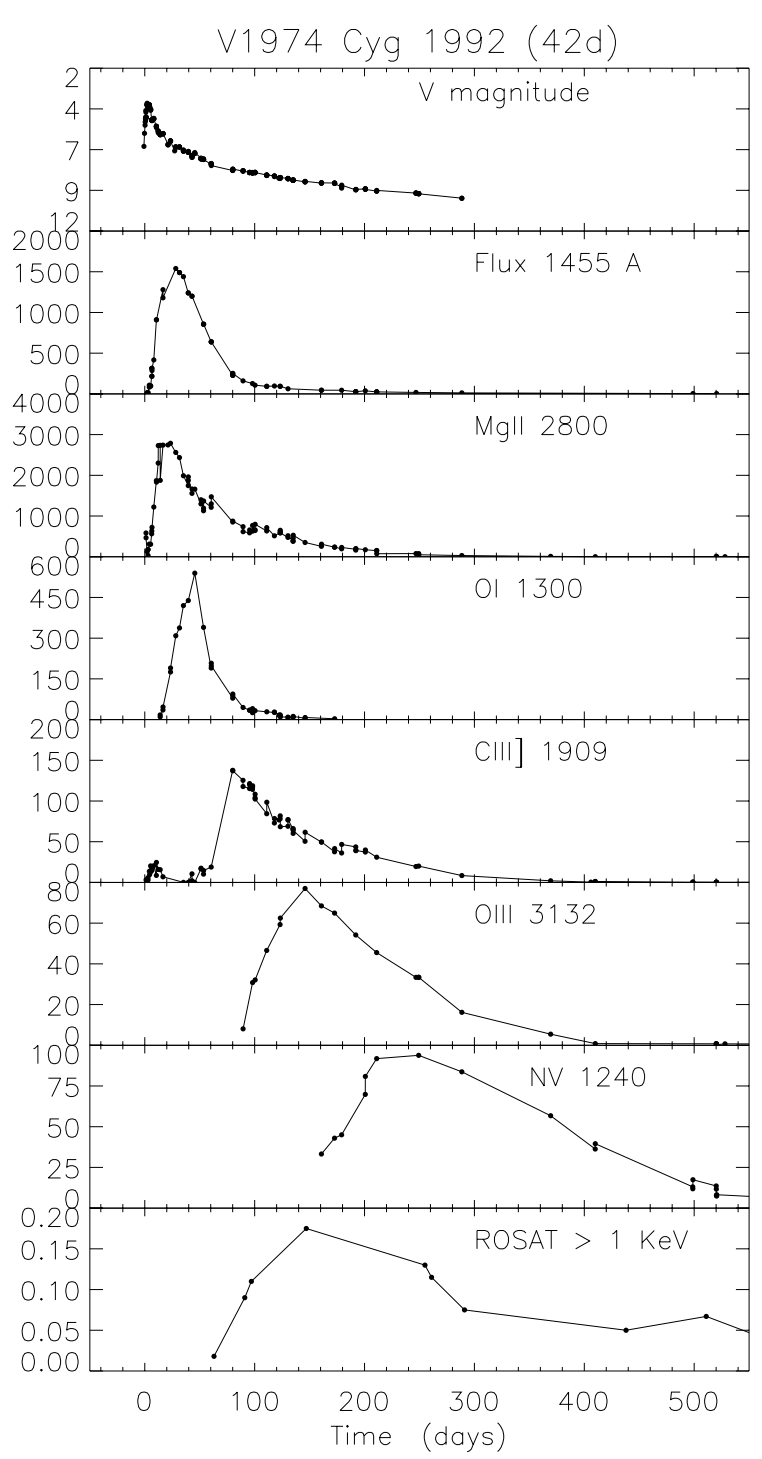

Fig. 1. Temporal evolution of the visual magnitude (from IAU circulars), of the observed fluxes in the $1455 \AA$ continuum and of some strong emission lines (from IUE low resolution spectra), and of the hard X-ray PSPC ROSAT emission (counts/s) (from Balman et al. 1998). IUE fluxes in the continuum and in the emission lines are in units of $10^{-12} \mathrm{erg} \mathrm{cm}^{-2} \mathrm{~s}^{-1} \mathrm{~A}^{-1}$ and of $10^{-12} \mathrm{erg} \mathrm{cm}^{-2} \mathrm{~s}^{-1}$, respectively.

day 143 for O III $3132 \AA$, and day 215 for N V $1240 \AA$. Note that the O III $3132 \AA$ line is one of the cascade transitions produced via the Bowen fluorescence excitation of O III by He II Lyman $\alpha$ (Saraph \& Seaton 1980), and can thus be used to track the He II $1640 \AA$ flux variations. (The He II line itself is not easily measurable at low resolution.) Similarly, the OI line is pumped by fluorescence of Lyman $\beta$ and therefore reflects the strength of the Lyman $\beta$ emission (see Cassatella et al. 2002, hereafter Paper I).

In Fig. 1 we show also, for further reference, the optical light curve (top; from the IAU Circulars) and the hard X-ray flux (bottom; from Krautter et al. 1996). Additional information on the UV spectral evolution of V1974 Cyg (and other novae) from $I U E$ low resolution spectra can be found in Paper I. 


\section{Spectral evolution of the P Cygni profiles and the UV emission lines}

In the following we discuss the spectroscopic changes of the following lines: Mg II $2800 \AA$, S II and Si II 1250-1265 C II $1335 \AA$, O I $1300 \AA$, Al III $1860 \AA$, Si IV $1400 \AA$, C IV $1550 \AA$, Lyman $\alpha$ and N V $1240 \AA$, which are shown in Figs. 2 and 3.

\subsection{P Cygni lines}

The UV spectra of V1974 Cyg generally show prominent $\mathrm{P}$ Cygni profiles in the resonance lines, especially at the earliest stages of the evolution. The strongest of these features is the Mg II 2796.35, 2803.52 $\AA$ doublet. It is useful to take it as a prototype for the evolution of the other P Cygni lines because it lies in a region of relatively low line blanketing and is thus easier to interpret.

\subsubsection{Mg || 2796.35, $2803.52 \AA$}

A sample of $\mathrm{Mg}$ II profiles starting from day 1.3 after discovery is given in Fig. 2a. A complete gallery of the Mg II profiles is shown in Shore et al. (1993).

In the first IUE high resolution spectrum of day 1.3, this line has a marked P Cygni profile consisting of strong emission and of very broad, shallow, and short-ward shifted absorption extending to an edge velocity $v_{\text {edge }}=3360 \mathrm{~km} \mathrm{~s}^{-1}$. The broad absorption component indicates that there is a large velocity gradient in the column to the observer.

The equivalent width of the emission component on day 1.3 (about $65 \AA$ ) exceeds that of the absorption by about $30 \%$. This indicates that the line is not formed by pure resonance scattering, but shows the presence of an extra source of excitation by electron collisions (Castor \& Lamers 1979; Lamers et al. 1987). This mechanism has been already suggested for the interpretation of the Mg II profile in V1668 Cyg 1978 (Cassatella et al. 1979).

The evolution of the Mg II line during the first two weeks can be described as a change from a P Cygni profile at day 1.3 into a pure emission profile at day 6 and later. The intensity ratio of the emission with respect to the absorption component increases from about 1.3 in the spectrum of day 1 to about 6 on day 14. The strengthening of the Mg II emission continues until day 21, when a maximum value is reached (see Fig. 1).

The broad absorption component of the P Cygni profile decreases in strength with time until, by day 16, it has vanished. When it gets weaker, it unveils the presence of much narrower violet-shifted absorption lines, which can be identified as belonging to the principal absorption system. (These lines are possibly present also before day 16 , but cannot be detected due to the depth of the P Cygni absorption component.) The radial velocity of these components increases with time (see Fig. 2a) reaching $-2020 \mathrm{~km} \mathrm{~s}^{-1}$ on day 135 , the last time these lines are seen (see Sect. 4.1). Note that the diffuse-enhanced components are not seen in Mg II (see Sect. 4).

From day 53 to day 211 one can detect, superimposed to the $\mathrm{Mg}$ II emission, narrow absorption components from the same doublet at a constant radial velocity of about $-600 \mathrm{~km} \mathrm{~s}^{-1}$ (see Fig. 2a). These components will be discussed in Sect. 4.2.

On day 192, the MgII line appears blended with the [Mg v] $2783 \AA$ emission, which on day 296 reaches about half the intensity of Mg II (see Fig. 2a). By this time the nova is far into the nebular phase. (The $[\mathrm{Mg} \mathrm{V}]$ emission may be present at earlier times, but is too faint to be detected.)

\subsubsection{S II uv1 and Si II uv4}

The SII uv1 and Si II uv4 transitions appear as emission-absorption features in the high resolution early spectra after day 16, when they start becoming detectable. As shown in Fig. 2b, only the principal system is present in these low excitation lines.

\subsubsection{C || 1334.53, $1335.68 \AA$}

The C II resonance doublet in Fig. 2c shows a marked P Cygni profile, although it is barely recognized in the earliest high resolution spectra due to the poor signal level and the heavy line blending. The emission component, like that of $\mathrm{Mg}$ II, is stronger than the absorption, and is flanked by narrow overlying absorptions which nearly disappear after day 35-40. The components from the $\mathrm{C}$ II principal and diffuse-enhanced systems are both detectable until day 53 , but their radial velocities cannot be determined accurately because of the small separation of the doublet lines (the radial velocities indicated in Fig. 2c are merely indicative and will not be used elsewhere).

\subsubsection{O । 1302.17, 1304.86, $1306.02 \AA$}

The evolution of the OI uv1 resonance triplet is shown in Fig. 2d. This line is blended with the Si II uv3 multiplet. Note the broad and asymmetric shape in the earliest spectra, and the apparent absence of the diffuse-enhanced absorptions from both ions. Since this line is formed by fluorescence (see Sect. 2), its profile cannot be considered as a classical P Cygni profile.

\subsubsection{Al III 1854.72, $1862.78 \AA$}

As shown in Fig. 3a, the behaviour of the Al III uv1 doublet is in some respects similar to that of $\mathrm{Mg}$ II: its shortward shifted absorption on day 1.3 has about the same edge velocity $\left(v_{\text {edge }} \approx\right.$ $3150 \mathrm{~km} \mathrm{~s}^{-1}$ ) as $\mathrm{Mg}$ II; the strength of the emission exceeds that of the absorption, their ratio being an increasing function of time; it appears as a strong feature already in the earliest spectra (this property is common to other Neon novae, see Shore et al. 1994).

The first radial velocity measurements of the principal system become possible on day 6.3 , and give a value of about $-1200 \mathrm{~km} \mathrm{~s}^{-1}$ (note that the principal system components were not detectable before day 12.3 in Mg II). Later on, on day 34, due to the very strong and wide absorption, the higher velocity components from the diffuse-enhanced system also appear, at a radial velocity of about $-2600 \mathrm{~km} \mathrm{~s}^{-1}$. The Al III 

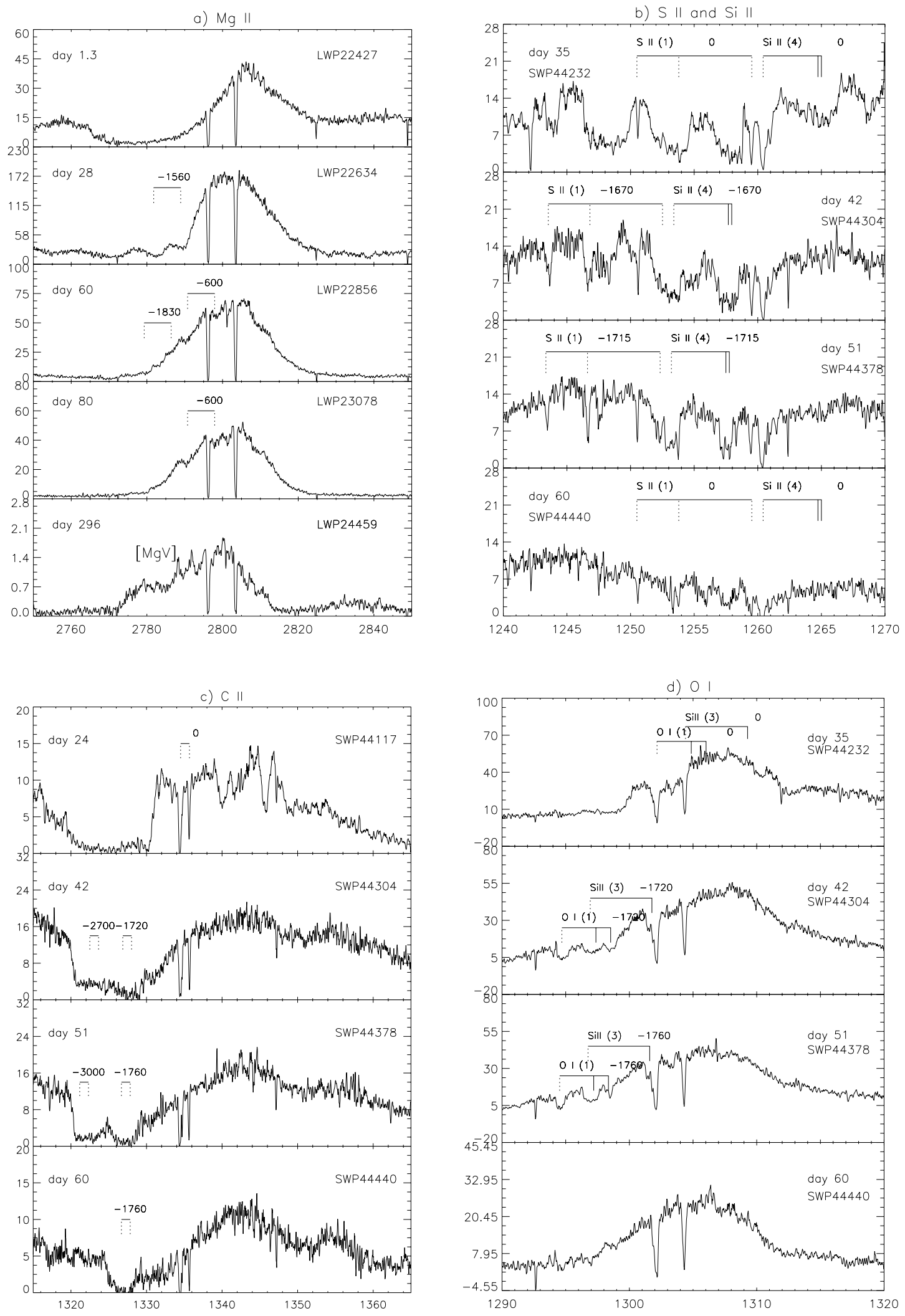

Fig. 2. Evolution of the line profiles in the region of Mg II, S II and Si II 1250-1265 ̊, C II $1335 \AA$, and O I $1300 \AA$. The $x$ and $y$ axes report wavelengths in $\AA$ and fluxes in units of $10^{-12} \mathrm{erg} \mathrm{cm}^{-2} \mathrm{~s}^{-1} \mathrm{~A}^{-1}$. The main absorption features and the corresponding radial velocities are indicated for the individual lines or multiplets ( 0 labels the laboratory wavelength, but sometimes is omitted). Transitions from the $0 \mathrm{eV}$ level are indicated as vertical dotted lines. 
a) $\mathrm{Al}$ III

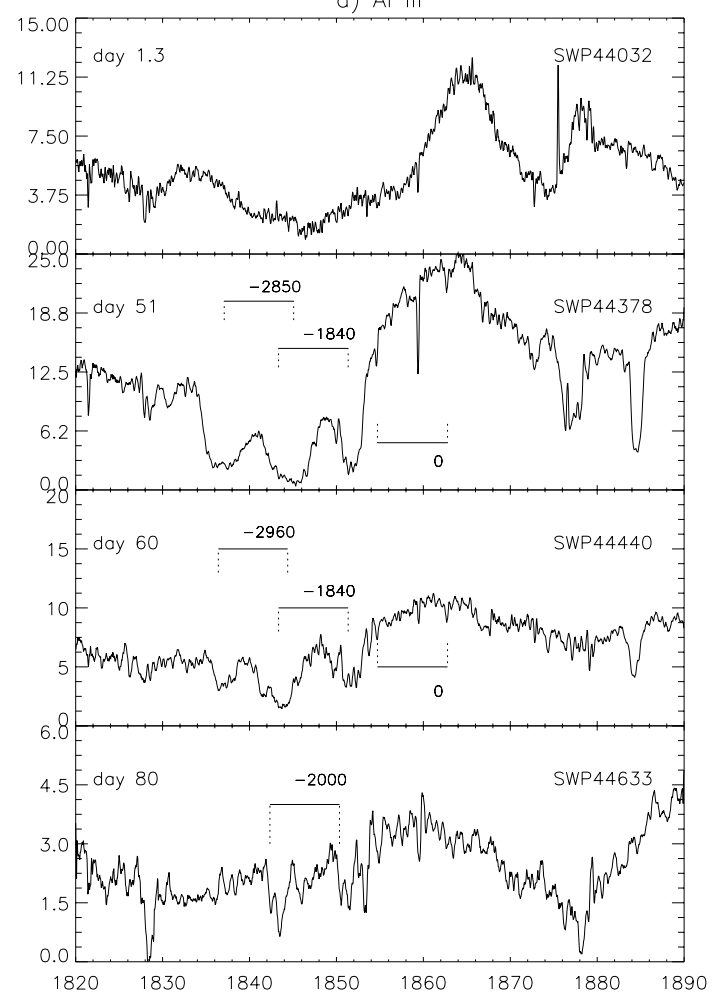

c) C IV

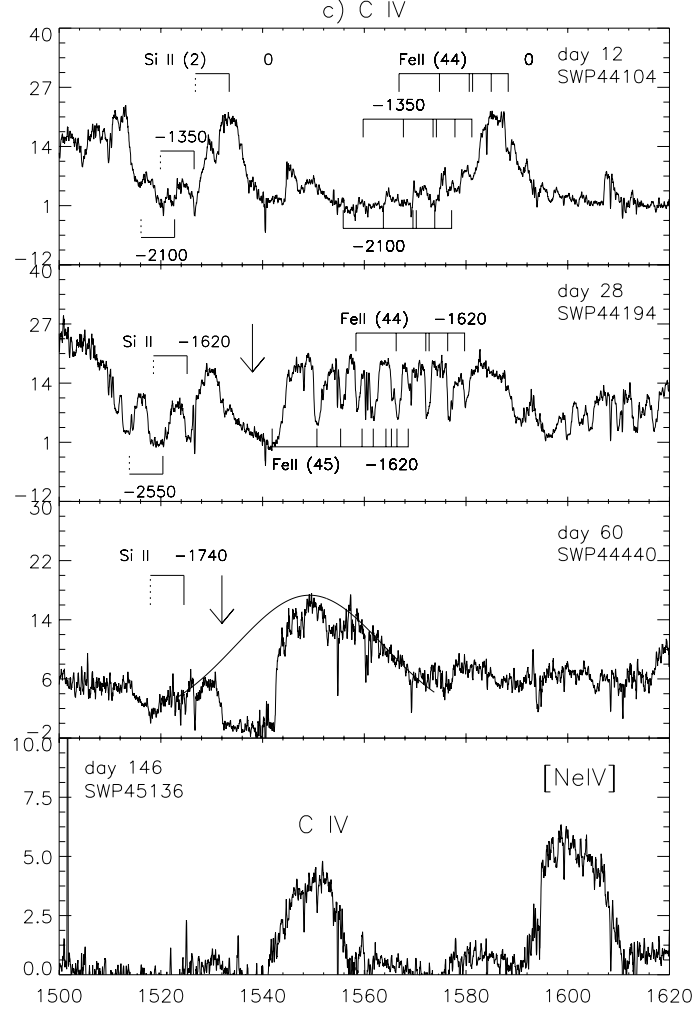

b) Si IV

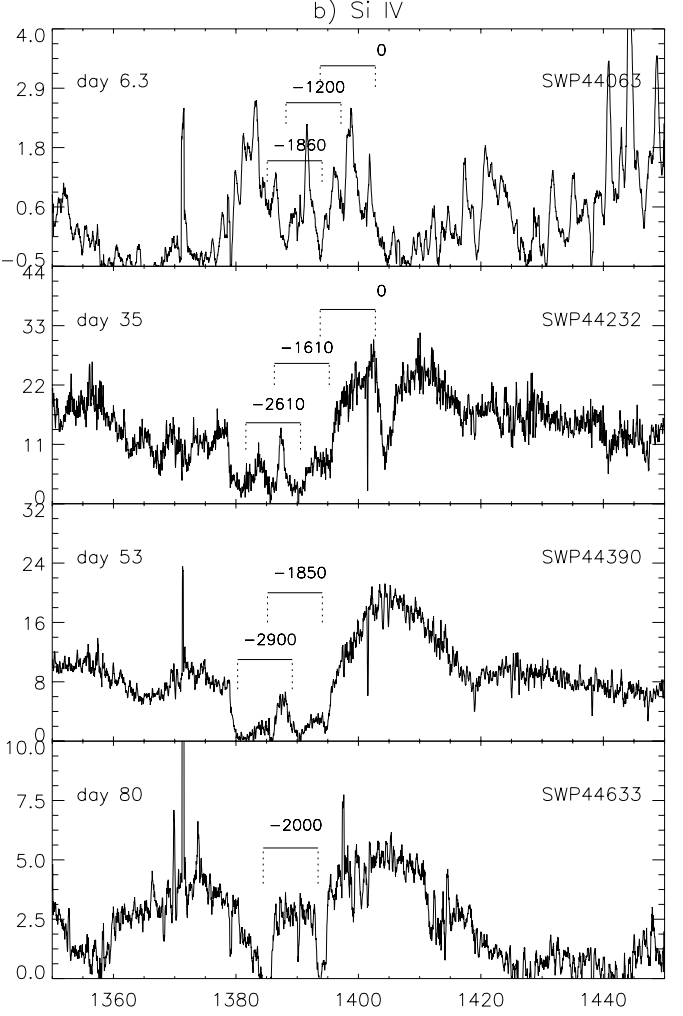

d) Lyman $\alpha$ and $N$ V

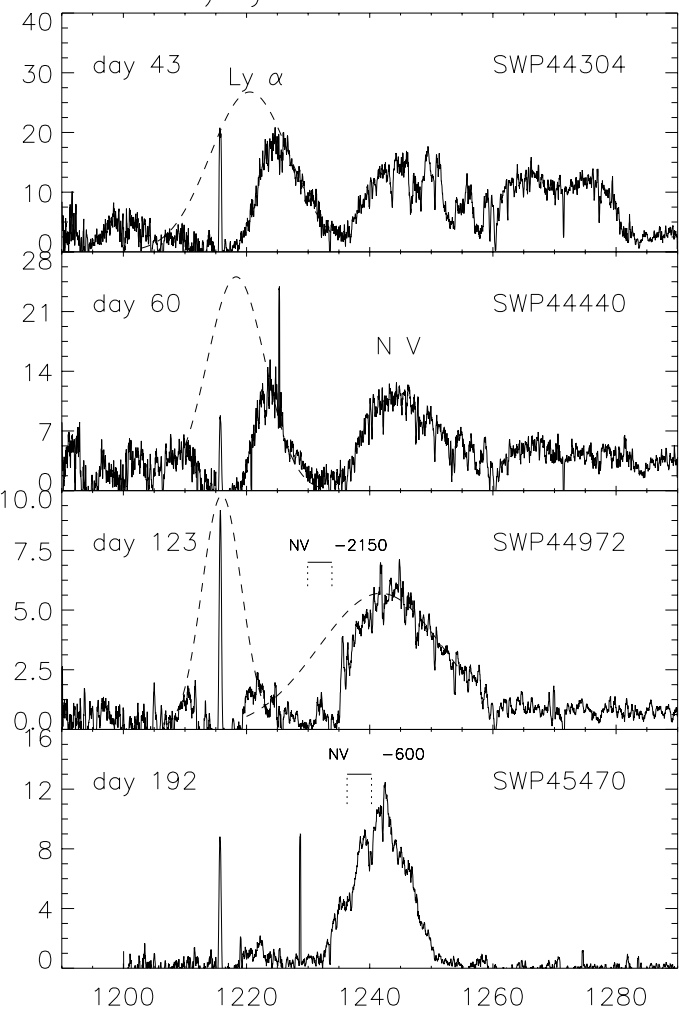

Fig. 3. Same as as in Fig. 2 for the lines of Al III, Si IV, C IV, and N V. See Fig. 6 for a complete identification of the C IV region on day 28 including the diffuse-enhanced absorption lines from Fe II. The position of the absorption through of the C IV line on day 28 and the wavelength corresponding to its edge velocity on day 60 are indicated with an arrow. Panel (d) shows the profiles of hydrogen Lyman $\alpha$ and $\mathrm{N} v$ at four epochs. To help in the identifications, we have indicated with a dashed line a rough approximation of these emissions with a Gaussian function. Note the progressive narrowing of the two emission lines, and the presence of absorption components from the principal system in the short wavelength wing of $\mathrm{N} \mathrm{V}$ on day 123 , and of low velocity components at $\approx-600 \mathrm{~km} \mathrm{~s}^{-1}$ on day 192 (see Sect. 3.1.8). 
diffuse-enhanced absorption system is clearly present until day 60 , but is certainly absent in the following observation of day 80 .

\subsubsection{Si Iv $1393.76,1402.77 \AA$}

As shown in Fig. 3b, the $1400 \AA$ A region is crowded with absorption lines. The shortward shifted absorption components from the principal and the diffuse-enhanced absorption systems of the Si IV resonance doublet begin to be seen clearly on day 6.3. Unlike Mg II and Al III, the P Cygni emission component of Si IV does not show up in the earliest spectra, due to the strong overlying absorption, but starts to emerge around day 23 , and becomes quite strong on day 53 . The diffuse-enhanced system is absent after day 60, the same as for the Al III line.

\subsubsection{Iv $1548.20,1550.77 \AA$}

The CIV resonance doublet is a very interesting feature for the way it gradually emerges and eventually shows up clearly in the spectra due to the decrease of overlying absorption by Fe II lines. Its evolution is shown in Fig. 3c. For a detailed identification of the Fe II lines on day 28 see Fig. 6. One can easily appreciate that the $1550 \AA$ A region is strongly blanketed by overlying absorption lines from the principal and diffuse-enhanced systems of Fe II uv44 and uv45, and of Si II uv2 (see also Sect. 4.1). The line blending and the degree of saturation is so severe that the identifications are very difficult in the earliest spectra (see for example the spectrum of day 12 shown in the top panel of thse figure). Despite this, it is possible to recognize the shortward shifted absorption trough of the C IV line already on day 12 and 28 (marked with an arrow in the latter spectrum). The complete sequence of IUE spectra, which cannot be shown here, indicates a progressive "clearing" of the overlying absorption until around day 40, when the P Cygni characteristics of C IV become evident. The overlying absorptions from Fe II multiplets uv44 and uv 45 become drastically reduced when, sometime after day 53 and before day 60 , the components from the diffuse-enhanced absorption system totally disappear. By day 146 the $\mathrm{C}$ IV feature has narrowed considerably and the [Ne IV] $1600 \AA$ forbidden line has become comparatively stronger, as shown in Fig. 3c. The [Ne IV] line reaches the maximum flux on day 173 .

\subsubsection{Nv 1238.80, $1242.78 \AA$}

The profile of the N v A resonance doublet is shown in Fig. 3d together with that of hydrogen Lyman $\alpha$. The latter line shows a broad central absorption dip of interstellar origin, discussed by Shore et al. (1993). The N V line begins to be clearly seen around day 47. By day 123 the short-ward shifted components of the principal system become measurable. Their position are indicated in the figure (note that the diffuse-enhanced components are not expected for this late date).

On day 123 one also starts to detect the presence of narrow shortward shifted absorption components of the $\mathrm{N} \mathrm{V}$ doublet at a radial velocity of $\approx-600 \mathrm{~km} \mathrm{~s}^{-1}$, superimposed on the broad emission. Contrary to the principal absorption lines, these

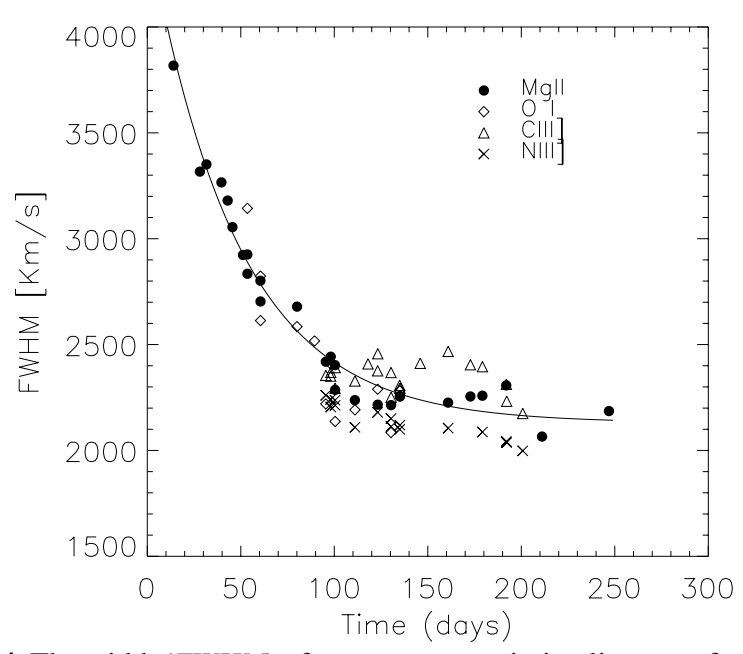

Fig. 4. The width $(F W H M)$ of some strong emission lines as a function of time. The full line represents an exponential fit to the Mg II data. The line widths were obtained by fitting the line profiles with a Gaussian function (the absorption components in the permitted lines were not included).

components, which are indicated in Fig. 3d for day 192, remain stable in wavelength until the last good quality short wavelength high resolution observations of day 201. See Sect. 4.2 for a further discussion.

\subsection{The widths of the emission lines}

We have measured the full width at half maximum (FWHM) of some of the strongest emission lines: Mg II $2800 \AA$, OI $1300 \AA$, C III] $1909 \AA$, and N III $1750 \AA$. The measured values, not corrected for the multiplet separation, are plotted as a function of time in Fig. 4 together with an exponential fit to the $\mathrm{Mg}$ II data. The figure shows an initial rapid decrease of the line widths from $\approx 3800 \mathrm{~km} \mathrm{~s}^{-1}$ in the first days, to $\approx 2450 \mathrm{~km} \mathrm{~s}^{-1}$ on day 90 . After this last date, the curve enters a near-plateau region characterized by a mean value $F W H M \approx 2140 \mathrm{~km} \mathrm{~s}^{-1}$. It is not possible to interpret this value in terms of expansion velocity of the nebular region because the multiplet components cannot be separated due to the large velocity field. Still, the exponential decrease of the emission line widths is a significant observational result in itself because it suggests formation in the inner and denser regions of the wind, where the expansion velocity is smaller.

We note that the width of the C III] $1909 \AA$ doublet is not easy to evaluate because it is partially blended with the Si III] $1893 \AA$ A doublet, as may be appreciated in the spectrum of day 80 (see Fig. 5). Shore et al. (1993) suggested a tentative identification of the $1893 \AA$ feature with Fe III with possibly a contribution from C III $1895 \AA$. Our main arguments in favour of the identification with Si III] are the following: a) the line was detected for the first time, at its nominal wavelength, on day 80 , i.e. at the same time as the $\mathrm{C}$ III] line (as it should, given the similar excitation conditions); b) the flux ratio C III]/Si III] increased with time (until the last high resolution observations on day 173), which is the expected consequence of the decreasing electron density in the ejecta (Nussbaumer \& Stencel 1987). 


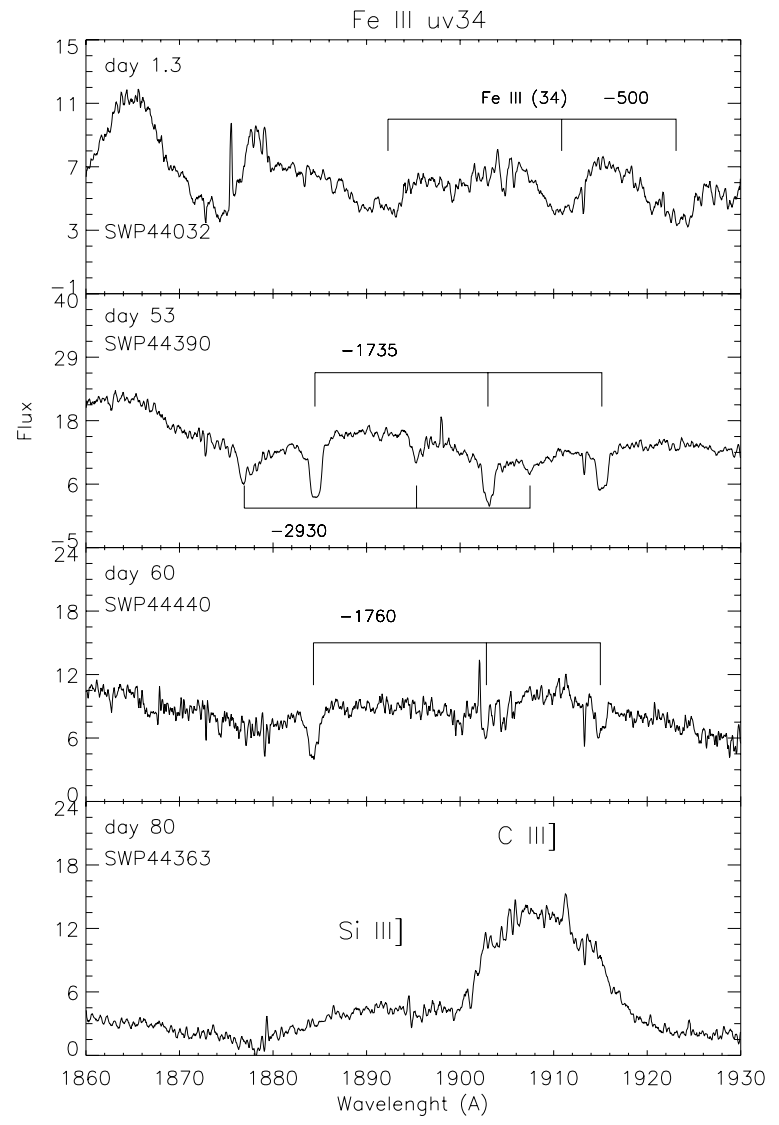

Fig. 5. Profile of the Fe III multiplet uv 34 at different dates. Note the disappearance of the diffuse-enhanced system by day 60 and the appearance of the $\mathrm{Si}$ III] and C III] doublets by day 80 . Fluxes are as in Fig. 2.

\section{The absorption line systems}

\subsection{Description and evolution of the absorption line systems}

Examples showing the identification of the principal system and of the diffuse-enhanced system at different dates and in different spectral regions are shown in Figs. 2, 3, 5 and 6.

The first IUE short wavelength high resolution spectrum was collected on day 1.3 , i.e. 2.2 days before visual maximum. Its complexity does not allow accurate radial velocity determinations to be made except in the 1890-1930 $\AA$ region where, due to the lower opacity, one can identify the principal system component of the Fe III uv34 multiplet lines at $1895.45,1914.05$ and $1926.3 \AA$ at a radial velocity of roughly $-500 \mathrm{~km} \mathrm{~s}^{-1}$ (see Fig. 5).

The principal system (low velocity) is present in all the permitted transitions studied. The diffuse-enhanced system (high velocity) is detected in the same transitions as the principal system (but only until day 60, as mentioned before) with the exception of the zero volt lines of the low ionization species of Mg II (see Fig. 2a), S II uv1, Si II uv4 (see Fig. 2b), and O I uv1 (Fig. 2d). This might be due to the low signal level in the wings of the corresponding emission line components. In any case, the presence of the diffuse-enhanced absorption system components from the excited levels of the O I $7773 \AA$ triplet and the Mg II $7876 \AA$ doublet has been reported in the ground spectra of V1974 Cyg obtained by Andrillat \& Houziaux (1993) during the early outburst. The higher excitation of the diffuseenhanced system compared to the principal system suggests that the diffuse-enhanced system is formed deeper inside the nova envelope (where the excitation conditions are higher) than the principal system.

The absorption components from the principal system are last seen in O I and Fe III on day 60, Mg II on day 135, and Si IV on day 146. Their disappearance is the result of a slow process caused by the increasing level of ionization and by the decrease of the column density due to the expansion.

The diffuse-enhanced system lines are absent in the spectra of day 1.3 , but present in the spectra of day 6.3 and later. They have vanished suddenly after day 60 for all the lines in which they had been detected earlier. The exact time cannot be determined due to the observational gap between day 53 and 60 . This point is discussed further in Sect. 4.3.3.

On day $42 \pm 7$, i.e. shortly before the disappearance of the diffuse-enhanced system, the O I emission reached a flux maximum (see Fig. 1). This event is a clear signature that the envelope started to be transparent to the $\mathrm{L} \beta$ radiation which pumps this transition (Paper I; Woodward et al. 1995), as demonstrated by the subsequent appearance of emission lines with increasing degree of ionization (see Sect. 2). Shortly after that time, a broad P Cygni profile from the C IV $1550 \AA$ A doublet popped up in the spectrum as a consequence of the decreased opacity in the envelope. On day 63 hard X-ray emission was detected for the first time with ROSAT (Krautter et al. 1996; Balman et al. 1998). Around day 70 important changes in the polarization properties were reported (Bjorkman et al. 1994). So we see that around day 53-70 significant changes occurred in the spectrum. These circumstances, all together, are discussed in Sect. 7.

We note finally that our principal system corresponds to what Chochol et al. (1997) referred to as the spherical component from the outer envelope. From day 2 to 62 these authors reported the presence of two additional absorption systems in the Mg II $2800 \AA$ doublet. One of them would correspond to the range of velocities displayed in other lines by the diffuseenhanced system. The other system has a radial velocity in the range $\approx-3790$ to $-4580 \mathrm{~km} \mathrm{~s}^{-1}$. Such high velocity absorption components have not been found by us in any of the spectral regions analysed. After a careful analysis of the $\mathrm{Mg}$ II profiles we cannot confirm the presence of these high velocity components. We believe that these lines are due to the diffuse-enhanced system from the Fe II uv32 lines $\lambda \lambda 2790.75$ and $2797.04 \AA$.

\subsection{The stable absorption components}

As anticipated in Sects. 3.1.1 and 3.1.8, there is evidence for stable and quite narrow absorption components from the Mg II and the N V doublets, which appear superimposed on the short wavelength wings of the corresponding emission lines, as shown in Figs. 2a and $3 \mathrm{~d}$ for $\mathrm{Mg} \mathrm{II}$ and $\mathrm{NV}$ on day 80 and 192, respectively. The peculiarity of these absorption lines is that their radial velocity, of about $-600 \mathrm{~km} \mathrm{~s}^{-1}$, is stable to 


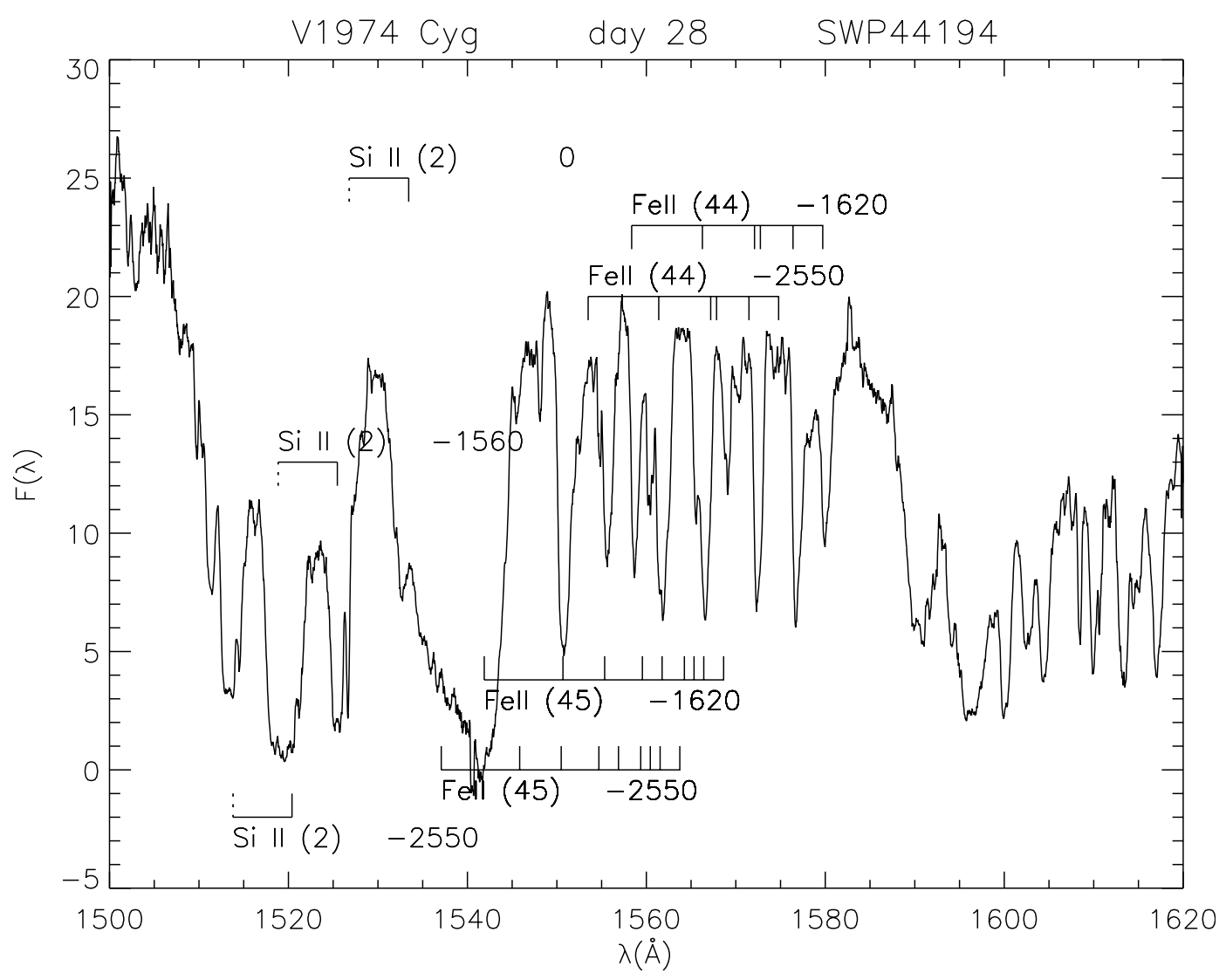

Fig. 6. Full line identifications in the C IV region on day 28. The figure shows how the CIV emission-absorption feature is affected by the overlying absorption from the principal and diffuse-enhanced systems of Fe II uv44-uv45 and Si II uv2. The unshifted Si II lines are interstellar. Fluxes are in the same units as in Fig. 2.

within about $20 \mathrm{~km} \mathrm{~s}^{-1}$ over a long period of time, i.e. from day 53 (when are first seen) to day 211 (last usable spectrum) for $\mathrm{Mg}$ II, and from day 123 (when are first seen) to day 201 (last usable spectrum) for NV. The Doppler widths of these absorption lines can be measured with some accuracy only when the underlying emission is strong enough. The values obtained are somewhat larger for $\mathrm{N} \mathrm{V}\left(F W H M \approx 180 \mathrm{~km} \mathrm{~s}^{-1}\right.$ in days 179-201) than for $\operatorname{Mg}$ II $\left(F W H M \approx 115 \mathrm{~km} \mathrm{~s}^{-1}\right.$ in days 95-211), while the equivalent width (only measurable for the $\mathrm{k}$ line components) is about the same $(\approx 0.15-0.20 \AA)$.

\subsection{The expansion velocity of the absorption systems}

Let us concentrate on the radial velocity variations of the principal and diffuse-enhanced systems in the 10 spectral regions shown in Figs. 2, 3, 5 and 6, where the most important line identifications are reported.

The radial velocity measurements have been carried out by fitting the absorption profile with a Gaussian function. The lines studied are: O I $1300 \AA$, Mg II $2800 \AA$, S II uv1, Fe II uv44, Fe II uv8, Fe III uv34, Al III uv1, Si IV uv1, and N v.

The radial velocity measurements are given in Tables 1 to 5 of the Appendix ${ }^{1}$. The measurements do not include the

\footnotetext{
1 Tables 1 to 5 of the Appendix are only available in electronic form at CDS.
}

C IV $1550 \AA$ A doublet because of the severe line blending at early dates, and the insufficient signal level at later dates.

\subsubsection{Accuracy of the radial velocity measurements}

González-Riestra et al. (2000) estimated the wavelength accuracy of IUE high resolution spectra by measuring the positions of interstellar lines in many well exposed $I U E$ spectra of standard stars, and found this to be $\pm 5 \mathrm{~km} \mathrm{~s}^{-1}$. Since our spectra are in general not equally well exposed as those of the standard stars, such a test has been repeated. To this purpose we have used the interstellar resonance lines of S II $1259.53 \AA$, C II 1335.68 А, and Mg II 2796.35 tained the following values: $-13 \pm 10$ (20 measurements), $-9 \pm$ $6 \mathrm{~km} \mathrm{~s}^{-1}$ (31 measurements) and $-20 \pm 5 \mathrm{~km} \mathrm{~s}^{-1}$ (46 measurements), respectively. The weighted mean of these measurements, $-15 \mathrm{~km} \mathrm{~s}^{-1}$, is taken to represent the accuracy of the relative radial velocity of the nova with respect to the interstellar medium, and the error $\pm 5 \mathrm{~km} \mathrm{~s}^{-1}$ (the same as found by González-Riestra et al. 2000), is the expected error in the radial velocity measurements of individual narrow lines in our spectra. However, we should consider that, among the absorption lines analyzed, only the ones from Fe II uv44-uv45 and of Fe III uv34 are about as narrow as the interstellar lines are. Indeed, the expected error in the velocities may be up to a 


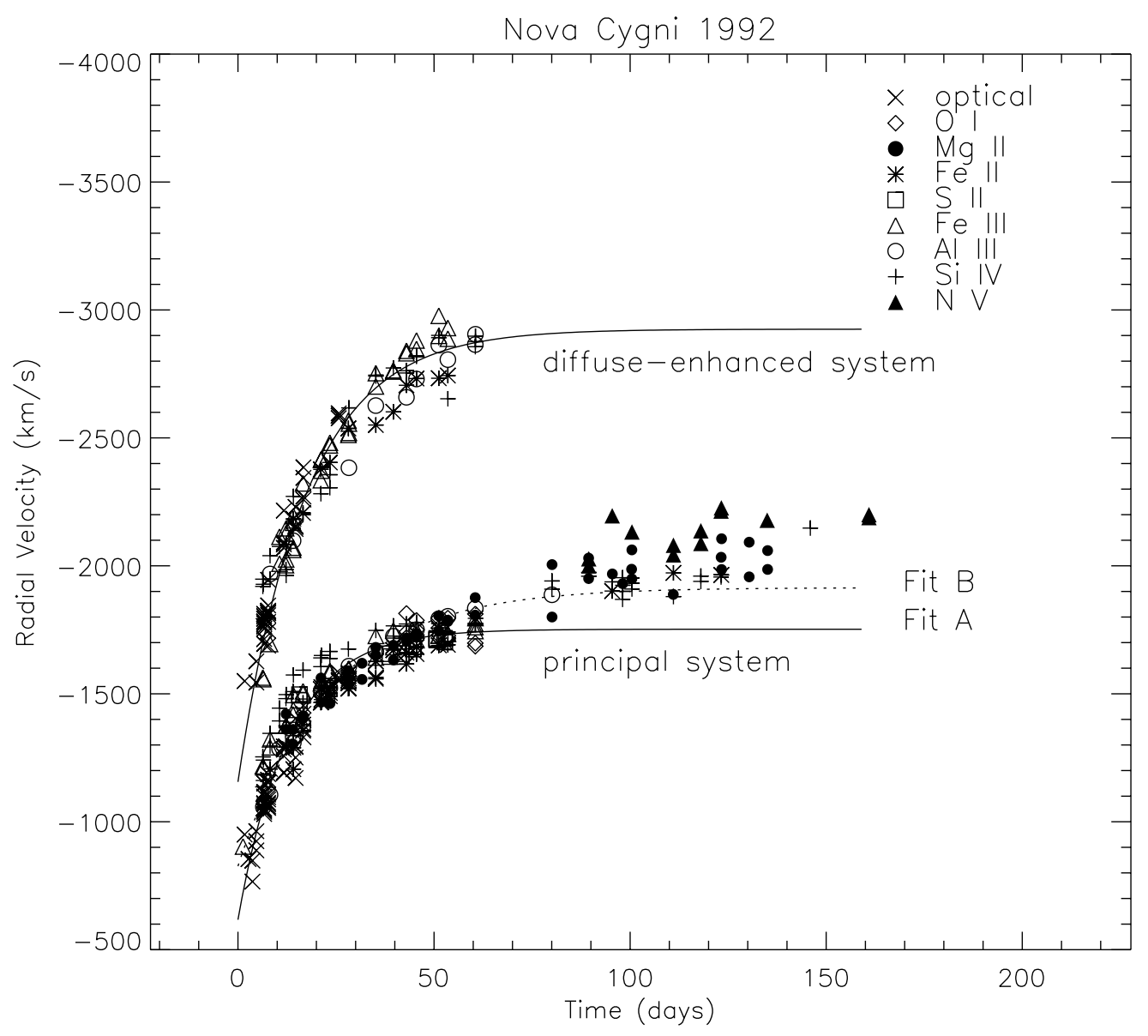

Fig. 7. Radial velocity of the principal and of the diffuse-enhanced absorption systems for the ions indicated in the upper right part of the figure. The velocity curves of the two systems have been fitted with the exponential law in Eq. (1) to obtain the coefficients in Table 1. Two fits are provided for the principal system: Fit $A$ is a better representation than $B$ for the fast rising part of the curve. Neither of the two representations is fully satisfactory for all the observations of the principal system. The figure suggests a sudden increase of the radial velocities of the principal system after day 53, with a mean value of $-2000 \pm 90 \mathrm{~km} \mathrm{~s}^{-1}$ (49 measurements) after that date. Note the large scatter in radial velocities after day 53 .

factor of four larger, depending on the line width and on the signal-to-noise ratio.

\subsubsection{The velocity law of the principal and diffuse-enhanced systems}

The measured radial velocities $v_{\mathrm{r}}(t)$ of the principal and diffuse-enhanced system are plotted in Fig. 7 as a function of time $t$ after the discovery. The figure also includes the radial velocity determinations from optical spectra (Annuk et al. 1993; Andrillat \& Houziaux 1993) which cover the earliest dates, when the strongest velocity gradient is observed.

Using a non-linear least squares algorithm, we have fitted the radial velocity curves with the following expression:

$v_{\mathrm{r}}(t)=-v_{\infty}+\left(v_{\infty}-v_{0}\right) \mathrm{e}^{-t / \tau}$.

The values of the parameters so obtained are reported in Table 1, together with the corresponding errors, the rms error of the fit and the number of measurements.

If the observed radial velocities $v_{\mathrm{r}}(t)$ reflect an outward expansion at a velocity $v(t)=-v_{\mathrm{r}}(t), \tau$ can be interpreted as a characteristic time related to the acceleration of the expanding matter, $v_{\infty}$ represents the asymptotic (i.e. terminal) velocity of the outflowing matter, and $v_{0}$ is the velocity at $t=0$.

Figure 7 shows that the radial velocity of the diffuseenhanced system until day 60 (when it definitively disappears) is fairly well represented by Eq. (1) with the coefficients in Table 1.

The situation is more complex for the principal system, due to the impossibility to represent with a unique fit both the rising part of the curve (corresponding to the earliest days) and the velocity "plateau" starting after day 80 , when the radial velocity curve becomes rather flat. We have therefore made two fits. The first, Fit A, was obtained from the observations made until day 60 (JD 2448 752); it fairly well represents this period, but it grossly underestimates the radial velocities in the "plateau" region which starts after day 80 . The second, Fit B, which includes all the measurements for the principal system, is a poorer representation than $F$ it $A$ for the earliest days, when the largest velocity variations are seen and, in addition, it clearly underestimates the observed values after day 80 . Both fits are shown in Fig. 7.

The failure to represent the radial velocity of the principal system with a unique law may be due to the limitation 
Table 1. Coefficients of the radial velocity law of the principal and diffuse-enhanced systems in Eq. (1).

\begin{tabular}{|c|c|c|c|c|c|c|}
\hline Absorption system & $v_{\infty}\left(\mathrm{km} \mathrm{s}^{-1}\right)$ & $\left(v_{\infty}-v_{0}\right)\left(\mathrm{km} \mathrm{s}^{-1}\right)$ & $\tau$ (days) & $\mathrm{rms}\left(\mathrm{km} \mathrm{s}^{-1}\right)$ & $n$ & Label \\
\hline Principal $t<60$ (days) & $1752.31 \pm 4.42$ & $1135.36 \pm 11.39$ & $13.139 \pm 0.259$ & 76 & 231 & A \\
\hline Principal (all data) & $1974.83 \pm 5.11$ & $1102.48 \pm 6.79$ & $27.580 \pm 0.447$ & 95 & 309 & B \\
\hline Diffuse (all data) & $2898.89 \pm 8.46$ & $1769.65 \pm 13.15$ & $16.396 \pm 0.302$ & 102 & 124 & \\
\hline
\end{tabular}

Note: Column 6 provides the number of measurements, inclusive 48 and 25 measurements for the principal and diffuse-enhanced systems, respectively, obtained from optical spectra (Annuk et al. 1993; Andrillat \& Houziaux 1993).
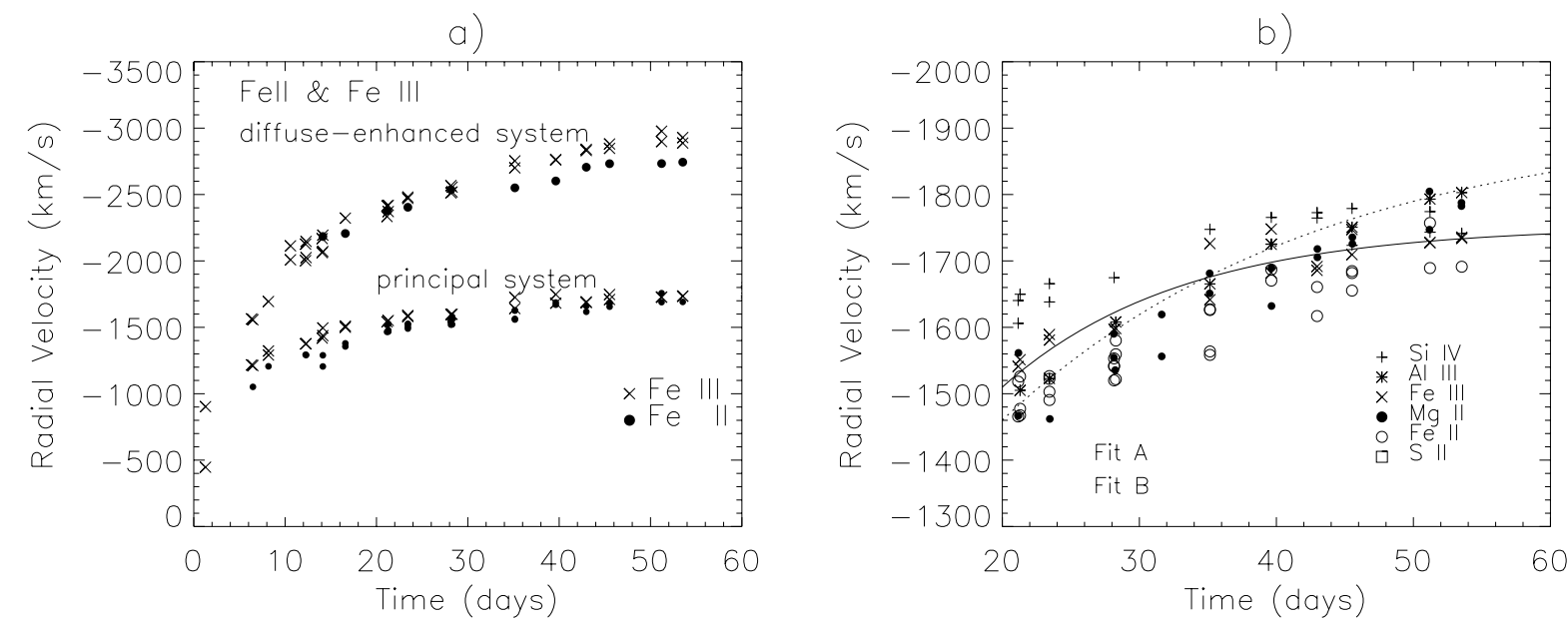

Fig. 8. Ionization stratification within the expanding regions. Panel a) shows the radial velocities of the Fe II (dots) and Fe III (crosses) lines from the principal and the diffuse-enhanced systems. Panel b) shows the data of Fig. 7, but for the principal system only, and only for a limited range of time and velocities. The figures indicate that the radial velocities are systematically more negative for the higher ionization species.

of the empirical representation of Eq. (1). An interesting alternative, which we adopt as working hypothesis, is that the velocity law of the principal system has actually changed after day 60. A careful inspection of the data in Fig. 7 suggests, in fact, that the radial velocity of the principal system has suffered from some sort of discontinuity after day 60, jumping from $-1760 \pm 47 \mathrm{~km} \mathrm{~s}^{-1}$ in days 53-60, to $-2000 \pm 90 \mathrm{~km} \mathrm{~s}^{-1}$ in the "plateau" phase after day 80 (from 27 and 49 measurements, respectively). In the following we will indicate with $v_{\text {plat }}^{\mathrm{ps}}=$ $2000 \mathrm{~km} \mathrm{~s}^{-1}$ the observed mean value of the expansion velocity of the principal system lines during the "plateau" phase.

As for the expansion velocity just before the jump, hereafter indicated with $v_{\infty}^{\mathrm{ps}}$, we have adopted the above observed value of $1760 \mathrm{~km} \mathrm{~s}^{-1}$ instead of the asymptotic value of $1752 \mathrm{~km} \mathrm{~s}^{-1} \mathrm{de}-$ rived from fitting the radial velocity data of the principal system before day 60 (see Table 1, Fit $A$ ). The velocity jump suffered by the principal system is then $\Delta v=v_{\text {plat }}^{\mathrm{ps}}-v_{\infty}^{\mathrm{ps}} \approx 240 \mathrm{~km} \mathrm{~s}^{-1}$. These values, together with the plateau velocity of the diffuseenhanced system $v_{\infty}^{\mathrm{ds}}=2899 \mathrm{~km} \mathrm{~s}^{-1}$ (see Table 1) will be used later in Sect. 5.

\subsubsection{Ionization stratification within the expanding envelope}

As shown in Sect. 3.1, the high ionization resonance lines, such as the $\mathrm{C}$ IV doublet, must be formed in regions which are deeper inside the envelope than the Fe II lines, because the Fe II lines absorb the broad C IV profile.

There is a second argument which throws light on another aspect of the stratification: radial velocities. Let us consider the radial velocity of lines of the same element, but in different ionization stages, such as Fe II and Fe III. The following lines, identified Figs. 3c and 5, were selected as being sufficiently strong and narrow:

- Principal system: 1584.95 and 1588.30 (Fe II uv44); 1608.45 and 1618.46 (Fe II uv8); 1895.46 and 1926.30 (Fe III uv34).

- Diffuse-enhanced system: 1895.46 and 1926.30 from Fe III uv34 and 1608.446 from Fe II uv8.

The radial velocity curves of the Fe II and Fe III lines shown in Fig. 8a not only confirm the presence of two expanding zones with different velocity regimes, but contain information about how the ionization is stratified within each region. In fact, it can be clearly seen that the Fe III radial velocities are systematically more negative than those of the lower excitation Fe II lines. If we assume that the ionization temperature decreases outward, as is well justified both by nova models (Kato \& Hachisu 1994; Bath \& Harkness 1989) and observations (Friedjung 1966b) it follows that, at a given time, the expansion velocity decreases outward within each region. Note that such stratification effects were also present in the data of Fig. 7. They become very clear when plotting the radial velocity curve of the principal system in a limited range of time and velocities, as done in Fig. 8b. 

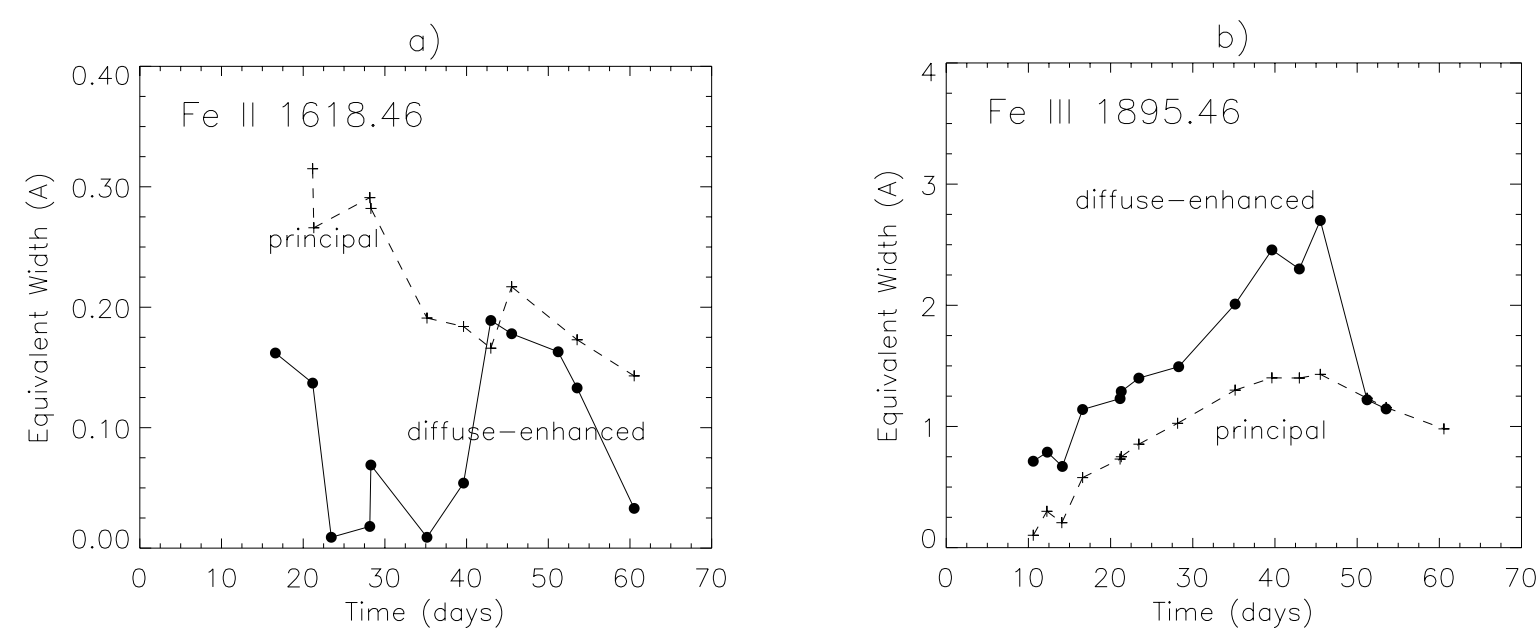

Fig. 9. The equivalent width of the absorption components from the principal and the diffuse-enhanced system as a function of time after outburst for: a) Fe II $1618.46 \AA$ and, b) Fe III $1895.46 \AA$. Up to day 40, the principal lines of Fe II are stronger than the diffuse-enhanced lines, whereas the reverse is true for the Fe III line (see Sect. 4.3.3). Note the sudden drop of the equivalent width for the Fe III diffuse-enhanced system on days 51-53. After day 53 the diffuse-enhanced component of the Fe III $1895.46 \AA$ absorption is no longer seen (Sect. 4).
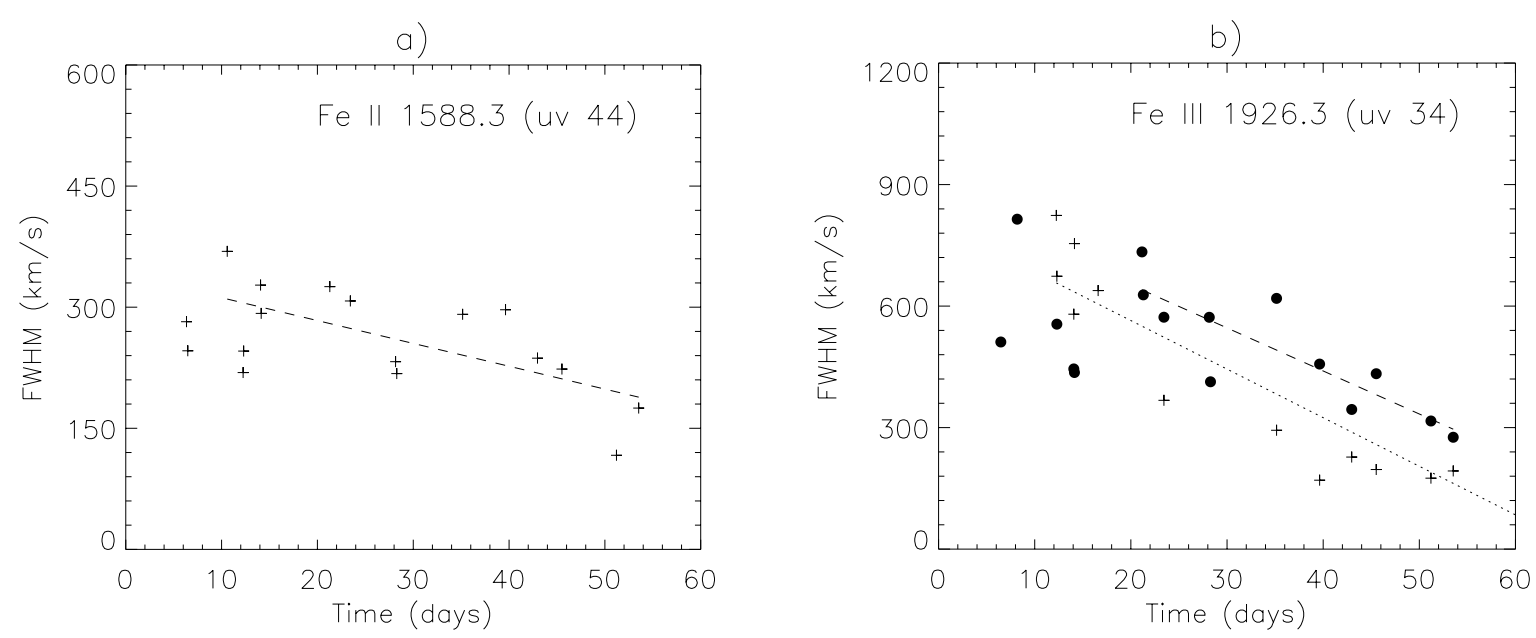

Fig. 10. The Doppler width ( $F W H M$ ) of the absorption components of a) Fe II $1588.3 \AA$ (principal system), and b) of Fe III $1926.3 \AA$ (principal and diffuse-enhanced systems) are plotted against time after the outburst. Linear regressions to the data of the principal system (crosses) and of the diffuse-enhanced system (dots) are indicated with a dashed line and a dotted line, respectively.

The presence of a velocity stratification within each of the expanding regions is what actually might cause the velocity spread at a given date $\left(\approx 70 \mathrm{~km} \mathrm{~s}^{-1}\right.$ for the principal system) to be substantially larger than the measurement errors (see Sect. 4.3.1).

A third way to study the ionization structure of the envelope is to analyse how the equivalent widths of the absorption systems vary with time for two transitions of the same element, but in different ionization stages. The results of this comparison are shown in Fig. 9 for the lines of a) Fe II the $1618.46 \AA$, and b) Fe III $1895.46 \AA$. If we restrict ourselves to the results before day 40 , we see that the Fe II line is significantly stronger in the principal system than in the diffuse-enhanced system while, on the contrary, the higher ionization Fe III line is stronger in the diffuse-enhanced system. These observations are clearly consistent with a model in which the diffuse-enhanced system is formed deeper inside the expanding envelope of the nova, where the degree of ionization is higher.

Finally, the stratification effects may be seen also in the Doppler widths $(F W H M)$ of the Fe II and Fe III lines from the principal and the diffuse systems. In Fig. 10 we show as a function of time the width of the Fe II $1588.3 \AA$ line from the principal system (the corresponding diffuse component cannot be used because of blending), and that of Fe III $1926.3 \AA$ from both the principal and diffuse systems. Indicative values of the line widths for the principal system component of Fe II $1588.3 \AA$ are: 300 and $180 \mathrm{~km} \mathrm{~s}^{-1}$ for days 15 and 55, respectively. In the same period, the widths of the Fe III $1926.3 \AA$ line decreases from $\approx 705$ to $280 \mathrm{~km} \mathrm{~s}^{-1}$ in the diffuse system and from $\approx 625$ to $144 \mathrm{~km} \mathrm{~s}^{-1}$ in the principal system. It can be clearly seen that a) the Fe III line is almost a factor of two broader than Fe II and b) the width of the 
diffuse-enhanced component of Fe III $1926.3 \AA$ is systematically larger than that of the principal system.

\section{Possible interpretations of the radial velocity curves}

\subsection{Non-spherical geometry of the outburst}

The case of non-spherical geometry of the ejecta has been investigated by Lloyd et al. (1993) to explain the increasing observational evidence for a bipolar structure in recurrent novae. In this framework, the high and low velocity absorption systems could arise from material ejected in the equatorial plane and along the polar axis, at different speeds. There is indeed unconfutable evidence for departures from spherical symmetry in the ejecta of V1974 Cyg 2-3 years after the outburst (Paresce et al. 1995; Shore et al. 1997). However, should such geometrical effects be applicable to novae in general, the wide spread of possible system inclination angles would cause the ratio between the expansion velocity of the diffuse-enhanced system and that of the principal system to vary in a wide range for different novae, in contrast with the observational evidence that it varies in a small range of values between 1.5 and 2 (McLaughlin 1960). In V1974 Cyg this ratio is about 1.6. For V1974 Cyg the major argument against a bi-directional interpretation of the two absorption line systems comes from the fact that both velocity systems are seen in absorption. This implies that the absorbing matter of both systems is in the line of sight from the emitting region (i.e. the close to the star) to the observer. This is difficult to reconcile with a model in which one of the systems comes from a disk and the other from polar ejections, unless both cover a large solid angle.

\subsection{Discrete shell ejections}

We propose a new model, described in detail in the next section, in which the principal and the diffuse-enhanced systems arise from two discrete expanding shells ejected in separate events. The principal system shell, or main shell, is impulsively ejected at the moment of the outburst, is located in the outermost expanding regions, and contains the bulk of the ejected matter. A following less massive impulsive ejection event gives rise to a second faster expanding shell, where the diffuse-enhanced system is formed. We provide below two arguments in favour of the above interpretation, which come from a constraint on the angular size of the nova on day 10 , and from the detection of X-ray emission on day 63.

a) The angular size and the distance of V1974 Cyg

According to the interferometric measurements of Quirrenbach et al. (1993) the angular radius of V1974 Cyg in hydrogen $\mathrm{H} \alpha$ on day 10 was $2.5 \pm 0.12$ mas. The distance to V1974 Cyg can be obtained by comparing this angular size determination with the distance reached by the principal system expanding shell, as predicted from the expansion law in Eq. (1).

The mean radius of the shell at time $t_{10}=10$ days can be obtained by integrating Eq. (1) over time. Using the appropriate constants for the principal system (Fit A from Table 1), and assuming that at the time of the outburst $t_{0}$ the radius of the shell is about $100 R_{\odot}$, one finds $r\left(t_{10}\right)=1.1 \times 10^{9} \mathrm{~km}$. This value, combined with the angular radius in hydrogen $\mathrm{H} \alpha$ on day 10 from Quirrenbach et al. (1993), leads to a distance of $2.9 \pm$ $0.2 \mathrm{kpc}$, which is in good agreement with the distance of 2.3 to $2.9 \mathrm{kpc}$ obtained by these authors by assuming a constant velocity in the range 1000 to $1300 \mathrm{~km} \mathrm{~s}^{-1}$. Our determination is also in agreement with the range of values, $\approx 2.2-3.4 \mathrm{kpc}$ obtained by Della Valle \& Livio (1995) from their upgraded maximum magnitude versus rate of decline relationship. We conclude that the velocity law of the principal system (see Eq. (1) and the coefficients of Fit $A$ in Table 1) is consistent with the extended source observed by Quirrenbach et al. (1993) on day 10 , if the principal absorption line system is formed in the outermost shell that contains most of the nova ejecta.

Our distance determination agrees also with the distance of 1.8 to $3.2 \mathrm{Kpc}$ given by Paresce et al. (1995) on the basis of FOC HST imaging in 1993-1995. According to Paresce et al. (1995) there is clear evidence from FOC observations that the expanding nebula was spherically symmetric in May 1993 and that it developed an elliptical ring shape by January 1994. Paresce et al. (1995) interpreted this morphological transformation, as well as the different expansion rates along the major and minor axes in terms of deposition of angular momentum by the $1.95 \mathrm{~h}$ period binary into the initially spherically symmetric nebula. The presence of an expanding ring around V1974 Cyg is also confirmed by the optical spatially resolved spectra obtained by Rosino et al. (1996) in 1994-1995, and by GHRS ultraviolet spatially resolved spectra obtained by Shore et al. (1997) in 1995.

\section{b) The origin of the hard X-ray emission}

Let us consider the hypothesis that the diffuse-enhanced system arises from regions which are located deeper inside the nova envelope and are expanding faster than those where the principal system is formed. We are interested in knowing if and when the fast moving diffuse system shell will overtake the principal system shell. This can be derived by integrating Eq. (1) over time. The implicit a priori assumption is that we are dealing with two separate shells, i.e. that the measured velocities reflect the velocity of the outflowing matter. As boundary conditions we impose that, at $t_{10}=10$ days, the shell that produces the principal system has a mean radius of about $r\left(t_{10}\right) \simeq 1.1 \times$ $10^{9} \mathrm{~km}$, as derived from the interferometric observations (see above). The mean radius of the shell that produces the diffuse system is not well known. If we assume that it is roughly $100 R_{\odot}$ on day 10 , and use the relevant constants in Table 1 , we find that the diffuse system shell will reach the principal system shell on day 30 at a radial distance of $3.6 \times 10^{9} \mathrm{~km}$ from the white dwarf centre. In reality, both the date of encounter and the radial distance should be considered as lower limits, since the encounter date is mainly determined by the low velocity in the early days, and the velocity law in Eq. (1) tends to overestimate the expansion velocities in the earliest days (see Sect. 4.3.2). Tests in this sense were made by making "local" fits (i.e. in restricted time intervals) to the radial velocity data 
in Fig. 7 and integrating the combined results. Keeping nearly the same boundary conditions as above, we find that the encounter took place at a later date, i.e. on day 42 , at a radial distance of $5.4 \times 10^{9} \mathrm{~km}$ from the white dwarf center. The large changes of the equivalent width of the diffuse-enhanced Fe II and Fe III lines which took place after day 40 (see Fig. 9) suggest that the latter date of encounter is more realistic than day 30 . We then assume that the two shells have started to merge together around day 42. Because of the finite thickness of the shells, this process will last until the less massive diffuse system shell is fully mixed with that of the supposedly more massive principal system. By this mechanism it is possible to account for the disappearance of the diffuse system between day 53 and 60, and the jump in the radial velocity at the "plateau" phase of the principal system.

With this hypothesis we can actually estimate the mass ratio of the principal and diffuse system shells $M_{\mathrm{p}} / M_{\mathrm{d}}$. In Sect. 4.3.2 we have discussed the possibility that the principal system shell, to reach the observed "plateau" value of $v_{\text {plat }}^{\mathrm{ps}}=$ $2000 \mathrm{~km} \mathrm{~s}^{-1}$, has accelerated after day 53 by $\Delta v=v_{\text {plat }}^{\mathrm{ps}}-v_{\infty}^{\mathrm{ps}} \approx$ $240 \mathrm{~km} \mathrm{~s}^{-1}$ from $v_{\infty}^{\mathrm{ps}}=1760 \mathrm{~km} \mathrm{~s}^{-1}$.

Suppose that such an acceleration is actually the result of a merging process of two shells with different masses and with initial and final velocities $v_{\infty}^{\mathrm{ds}}=2899 \mathrm{~km} \mathrm{~s}^{-1}$ and $v_{\text {plat }}^{\mathrm{ps}}$, respectively. We can then evaluate the mass ratio from the momentum conservation equation as $M_{\mathrm{p}} / M_{\mathrm{d}}=\left(v_{\infty}^{\mathrm{ds}}-v_{\mathrm{plat}}^{\mathrm{ps}}\right) / \Delta v$. With the quoted values for the velocities we find that the principal system shell is roughly 4 times more massive than that of the diffuse system.

If the disappearance of the diffuse-enhanced system is due to the formation of a shock front, a hot plasma is produced, whose temperature can be evaluated from the velocity difference $\Delta v_{\mathrm{pd}}=v_{\infty}^{\mathrm{ds}}-v_{\infty}^{\mathrm{ps}}$ between the asymptotic velocities of the two systems as

$T_{\text {shock }}=\frac{3}{16} \frac{\mu m_{\mathrm{H}}\left(\Delta v_{\mathrm{pd}}\right)^{2}}{\kappa} \approx 12.1\left(\frac{\Delta v_{\mathrm{pd}}}{100 \mathrm{~km} \mathrm{~s}^{-1}}\right)^{2} \mathrm{eV}$

(see Lamers \& Cassinelli 1999). With $v_{\infty}^{\mathrm{ds}}=2899 \mathrm{~km} \mathrm{~s}^{-1}$ (see Table 1) and $v_{\infty}^{\mathrm{ps}}=1760 \mathrm{~km} \mathrm{~s}^{-1}$ (Sect. 4.3.2) we obtain a shock temperature $T_{\text {shock }} \approx 1.6 \mathrm{keV}$. This value is lower than the value of $\approx 10$ and $5 \mathrm{keV}$ observed by Balman et al. (1998) on day 63 and 91 , respectively, but is close to the nearly constant temperature of the X-ray hard emission component reported by Balman et al. (1998) for days 97 to 653. We can reasonably conclude that the hard X-ray emission from V1974 Cyg is most likely due to shock interaction of the diffuse-enhanced system with the principal system matter.

\section{Towards an empirical model}

In this section we summarize the important observational results of this paper and propose an empirical model to explain the observations.

\subsection{The observed properties}

1. The UV spectrum at early phases (day $\lesssim 14$ for Mg II and later on for higher excitation lines), is characterized by $\mathrm{P}$ Cygni profiles in the major UV resonance lines, with very high edge velocities of about $3400 \mathrm{~km} \mathrm{~s}^{-1}$. The P Cygni profiles gradually change into full, approximately symmetric emission lines (see Sect. 3.1). This suggests that line formation progressively shifts toward the inner and denser regions of the pseudo-photosphere.

2. The width $(F W H M)$ of the emission lines decreases rapidly with time from about $3800 \mathrm{~km} \mathrm{~s}^{-1}$ on day 14 , to $2300-2400 \mathrm{~km} \mathrm{~s}^{-1}$ on day 100 . It then decreases at a much slower rate down to $2100 \mathrm{~km} \mathrm{~s}^{-1}$ on day 250 . When the emission dominates the P Cygni profiles (after about day 20 for $\mathrm{Mg}$ II), the density in the wind must be high (see Sect. 3.2). So the decreasing width of the emission components suggests that the line-emitting layers move deeper into the accelerating layers of the wind, or that the wind velocity is decreasing with time (but there is no evidence for this in the present case).

3. The time of maximum emission depends strongly on the ionization stage: $\mathrm{Mg}$ II reaches its maximum on day 21, O I $\lambda 1300 \AA$ (which via fluorescence reflects the strength of Lyman $\beta$ ) peaks at day $42, \mathrm{C}$ III] on day 77 , O III on day 143, and N V on day 215 (see Sect. 2). This shows that the degree of ionization in the wind increases with time as the central source gets hotter.

4. The IUE observations show that, including the very first days, low excitation narrow absorption lines can be detected superimposed on the P Cygni profiles or the emission profiles (see Sect. 3). This indicates that the emission lines are formed inside the region where the absorption components are formed.

5. Two velocity systems of absorption lines can be recognized: the principal system and the diffuse-enhanced system. At any time, the expansion velocity of the diffuseenhanced system is higher than that of the principal system by about 600 to $1000 \mathrm{~km} \mathrm{~s}^{-1}$. The expansion velocity of both systems increases with time according to Eq. (1), exponentially reaching a velocity of $\approx 1750 \mathrm{~km} \mathrm{~s}^{-1}$ and $\approx 2900 \mathrm{~km} \mathrm{~s}^{-1}$, respectively (see Table 1 ).

6. The principal system lines are present in the spectra of day 1.3. The diffuse-enhanced system lines are absent on day 1.3 but they are present in the spectra of day 6.3. The optical spectra by Andrillat \& Houziaux (1993) show that the diffuse-enhanced lines are first seen at about day 3.3 and are already strong on day 5 (unfortunately we do not have UV-spectra in this period). This shows that the P Cygni profiles that are observed on day 1.3 mark a phase in between the formation of the principal and of the diffuse-enhanced system lines.

7. The width $(F W H M)$ of the absorption components of both the principal and the diffuse systems decreases with time, but the diffuse system lines are generally broader than the principal system lines (see Fig. 10).

8. Within both regions that form the principal system and the diffuse-enhanced system lines, the higher ionization lines 
have a slightly higher expansion velocity. The velocity difference is of the order of $200 \mathrm{~km} \mathrm{~s}^{-1}$ only. This suggests that within each of these two regions, at any time, the higher velocity layers are deeper than the lower velocity layers.

9. After day 60 the diffuse-enhanced system lines disappear suddenly, and shortly thereafter the principal system lines show a discontinuous increase in velocity of $\approx 240 \mathrm{~km} \mathrm{~s}^{-1}$. Around the same period, other significant changes in the spectrum occur: hard X-rays are observed for the first time, and the degree of polarization changes. This has been explained in Sect. 5.2, by means of an encounter/collision and final merging of the two shells where the two absorption line systems are formed.

10. From about day 53 to 211 , when there is enough signal in the short wavelength emission wing of the Mg II emission, narrow shortward-shifted absorption components are seen superimposed on the emission at a stable radial velocity of about $-600 \mathrm{~km} \mathrm{~s}^{-1}$ (Fig. 2a). The same stable absorption components at $-600 \mathrm{~km} \mathrm{~s}^{-1}$ are also seen in the $\mathrm{N} \mathrm{V}$ doublet from day 123 to 201, but are best detected near the latest dates, when the underlying doublet emission has become strong enough (see Fig. 3d). These narrow and stable absorption lines are probably formed at a very large distance from the star from matter ejected in the WD-wind before the nova eruption, not yet perturbed by it. Their later appearance in $\mathrm{N} \mathrm{V}$ than in $\mathrm{Mg}$ II is a signature of the increasing degree of ionization in the pre-nova wind.

\subsection{A multi-phase model}

To explain the observed characteristics, we consider a schematic dynamical model in which mass loss has gone trough five phases. Each mass loss phase is responsible for the occurrence of certain spectroscopic features. In a time sequence of events, these phases are:

I The pre-nova wind phase. A low mass loss wind from the white dwarf sets in with a velocity of about $-600 \mathrm{~km} \mathrm{~s}^{-1}$ long before the outburst.

II The main ejection phase. This phase probably starts about 3 days before the discovery time (see introduction) and lasts a very short time ( $\lesssim 1$ day). It is characterized by a high mass loss rate, and by an initial wind velocity of at least $\approx 500 \mathrm{~km} \mathrm{~s}^{-1}$ at day zero. This high mass loss phase produces the main shell that contains most of the ejected matter and gives rise to the principal absorption line system. During this phase, the ejection velocity increases with time, probably due to a decreasing mass loss rate, as e.g. predicted in the optically thick nova ejection models by Kato \& Hachisu (1994).

III An intermediate wind phase with a lower mass loss rate but with a very high outflow velocity. It is responsible for the P Cygni profiles seen on day 1.3.

IV A second ejection phase of high mass loss rate. It takes place at some stage between day 3 and 5 and lasts a very short time ( $\$ 1$ day). The mass loss rate is lower and the velocity is higher than during the first ejection phase. Also during this phase the wind velocity increases with time, possibly accompanied by a decreasing mass loss rate. The resulting shell gives rise to the absorption components of the diffuse system.

$\mathrm{V}$ A high velocity wind phase of relatively low mass loss rate. It lasts relatively long, about $10^{2}$ days at least. The P Cygni profiles and the emission lines are produced in this wind. This phase represents the natural, smooth continuation of phase III, but is characterized by a lower mass loss rate and ejection velocity.

The above time sequence of mass loss phases (or episodes in the case of the main and second ejections) gives rise to a highly stratified envelope, whose structure changes with time. To illustrate these changes we have sketched in Fig. 11 the radial profile in density and velocity for day 10 and day 60 . The structure of the envelope around day 10 is rather complex. From inside to outside we find: the high velocity wind (which is accelerated in a optically thick inner region), the second shell (giving rise to the diffuse system), an intershell region between the second shell and the main shell (we have little information on this shell except that its column density must be so low that it does not produce detectable absorption components), the massive main shell, and the pre-nova white dwarf wind. By day 60 the second shell has merged with the main shell and the wind is no longer optically thick.

We stress that the velocity decreases outwards in both the main shell and the second shell. This is not due to a deceleration of the wind (which would be impossible, because the gravity of the white dwarf is not strong enough to decelerate the wind once it has moved away more than about $10 R_{\odot}$ or about $10^{3} R_{\mathrm{WD}}$ ), but is due to the fact that the ejection velocity increased with time, probably in step with a decrease of the mass loss rate.

\subsection{The structure and evolution of the envelope}

The evolution of the individual components of the nova envelope and the observational consequences are better understood if the innermost region, which provides the ionizing flux to the rest of the envelope, is discussed first. Then, going from the inner to the outer regions, the evolution can be described as follows:

The high velocity wind sets in after the other mass loss phases. It gives rise to the emission profiles that are seen as prominent features after about day 6 . Up to roughly day 14 the mass loss rate is so high that this wind is optically thick in the UV continuum, so that the pseudo-photosphere lies within the wind. As the mass loss rate decreases, the wind becomes optically thin in the continuum, and the lower layers of the pseudo photosphere move inwards. This has two effects: (1) the effective temperature increases as the effective radius of the star decreases, and (2) radiation from the inner layers of the wind, where the velocity is smaller and the density is higher than outside, becomes detectable. This explains the decreasing width of the emission components (Figs. 2, 3 and 4), as well as the increase in the degree of ionization in the wind with the higher ionization stages peaking at later times than lower ionization stages (Fig. 1). 


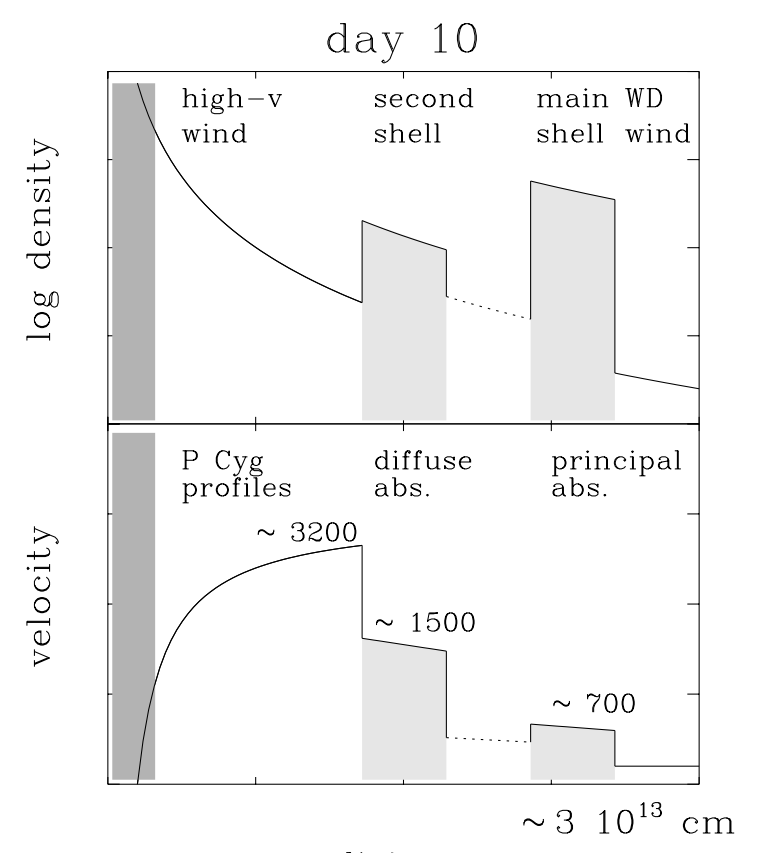

distance

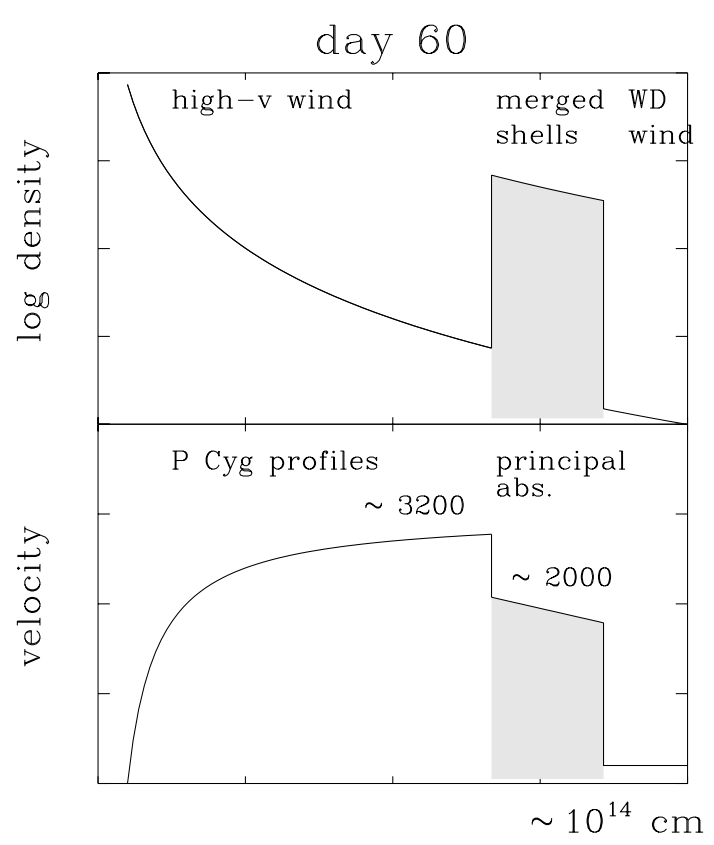

distance

Fig. 11. A sketch of the density structure (top) and velocity structure (bottom) of an empirical multi-phase wind model at about day 10 , and day 60 described in Sect. 6.2. The distance scale is only approximate. The different zones are indicated as well as the approximate velocities. On day 10 (left hand panel), the two absorption line systems are formed in the two shells (light grey). In this early phase the mass loss rate of the wind is so high that the pseudophotosphere is located inside the wind. Radiation from the deeper high density layers of the wind (dark grey) does not escape. On day 60 (right hand panel) the high velocity wind is no longer optically thick in the continuum, so the emission lines from the deepest wind layers can be observed. The second and the main shell have merged into a single shell (light grey). The mass loss rate has decreased so that the inner part of the wind is no longer optically thick.

The second ejection phase at around day 3 to 5 produces the second shell that has an initial velocity of about $1500 \mathrm{~km} \mathrm{~s}^{-1}$ or less (Fig. 7). The absorption lines of the diffuse system are formed in this shell. During the ejection, which lasts a short time, the ejection velocity slightly increases with time, which results in an outward decreasing velocity. This explains why the higher ionization absorption lines show a slightly more negative Doppler velocity than e.g. the Fe II lines (Fig. 8). As the star gets hotter as a consequence of the receding pseudo-photosphere, its increasing UV flux accelerates the shell by radiation pressure in the very numerous UV absorption lines, similar to the radiative acceleration of line-driven winds. This explains the increase in the radial velocity of the diffuse system up to a velocity of $\approx-2800 \mathrm{~km} \mathrm{~s}^{-1}$ near day 60 (Fig. 7). As the shell moves outward, its column density decreases and the absorption lines get weaker (Fig. 9) and narrower (Fig. 10). At the same time, the degree of ionization increases because the star gets hotter. This explains why the equivalent width of the Fe II lines decreases, whereas the Fe III absorption lines first get stronger up to day 40 and then get weaker (Fig. 9). The decrease of the column density with time has another observable effect. The region where the absorption lines are formed moves deeper into the shell. This implies that the absorption occurs over a region with a smaller velocity range. This explains the observed narrowing of the absorption lines (Fig. 10).
The main shell is ejected at a velocity of less than of $700 \mathrm{~km} \mathrm{~s}^{-1}$ (Fig. 7) about 3 days before the discovery. The absorption lines of the principal system are formed in this shell. There is a small negative velocity gradient, which explains the difference in Doppler velocity of the absorption lines of different ionization stages (Fig. 8). Radiation pressure accelerates this shell in a similar way as the shell where the diffuse absorption lines are formed, and the main shell reaches a velocity of about $-1800 \mathrm{~km} \mathrm{~s}^{-1}$ near day 60 . The degree of ionization slowly changes with time (Fig. 9) in the same way as in the second shell, and the absorption lines get narrower (see Fig. 10) for the same reason. The velocity difference between this shell and the faster moving second shell inside leads to a merging of the two shells around day 60, as explained in Sect. 5.2. This merging leads to the disappearance of the diffuse system absorption lines after day 60 , the jump in velocity of the principal system absorption lines, of about $240 \mathrm{~km} \mathrm{~s}^{-1}$, which occurs between day 60 and 80, and the detection of hard X-rays around that time. This situation at about day 60 is sketched in Fig. 11. The remaining shell will be overtaken by material from the fast wind. However, the density contrast between the main shell and the fast wind may be so large that the dynamic acceleration of the shell will take a long time. It will probably lead to afragmentation, as is observed in old nova shells (Slavin et al. 1995).

The material ejected by the white dwarf during the prenova wind phase is little disturbed during the first few 
hundred days. This material produces the very stable, weak and narrow absorption lines seen at $-600 \mathrm{~km} \mathrm{~s}^{-1}$ on top of the $\mathrm{Mg}$ II and the $\mathrm{N}$ V doublets (see Fig. 5 of Sect. 3.1.8 and Fig. 2 of Sect. 3.1.1).

\subsection{Interaction between the different wind phases?}

We notice that the overall outward decrease of the expansion velocity in the different wind regions will lead to the wind layers catching up with their neighbours. Yet there is little evidence for merging of the layers, except for the second shell merging with the main shell. For instance, the fast wind with its velocity of about $3500 \mathrm{~km} \mathrm{~s}^{-1}$ observed on day 1.3, i.e. in between the two shell ejections, should catch up with the main shell with its initial velocity of about $700 \mathrm{~km} \mathrm{~s}^{-1}$, increasing to $1500 \mathrm{~km} \mathrm{~s}^{-1}$, in about 10 to 15 days, if the main shell was ejected 3 days before the fast wind started. The lack of a clear sign of the interaction suggests a large density contrast between the wind and the main shell.

One of the most significant results of this study is the simultaneous increase in velocity of the main shell and the second shell. We have explained this in terms of radiative acceleration by the star due to its increasing UV flux. Could the acceleration be due to dynamic interaction (momentum transfer) of the different shells? This seems unlikely, given the fact that the acceleration of both shells up to day 60 is remarkably similar (Fig. 7). If the main shell was accelerated by the interaction with the second shell, and the second shell by interaction with the fast wind, the similar acceleration would require a finetuning between the momentum increase of the two shells by the two interaction zones, which seems unlikely. On the other hand, if the acceleration of the two shells is due to radiation pressure, the similar acceleration is simply the result of radiation pressure by optically thin lines in both shells. For radiation pressure in optically thin lines the radiative force per unit mass, and thus the acceleration, is proportional to the flux and independent of the density. So shells with different densities, but with rather similar ionization conditions, will experience the same acceleration. Still, the shell closer to the star will experience a slightly larger acceleration because of the larger incoming flux. The different radial distance of the shells explains why the second shell, which is closer to the star than the main shell, experiences a slightly larger acceleration.

An interesting question is how the above interaction would affect the velocity field of the pre-maximum matter. The only trace we have of the pre-nova wind is the presence of narrow and stable components of the $\mathrm{N} \mathrm{V}$ doublet at $-600 \mathrm{~km} \mathrm{~s}^{-1}$ appearing superimposed on the doublet emission. The stability in velocity and equivalent width of these narrow components between day 123 and 192 is not suggestive of formation by dynamical interaction, but rather supports the idea that they are formed by photoionization in a low density region far away from the nova.

\section{Discussion and conclusions}

The present investigation, based on high resolution ultraviolet spectroscopy of V1974 Cyg has led to an empirical model in which the object goes through different mass loss phases. The major results of this study are:

(1) In the earliest days the high velocity wind is optically thick so that the pseudo-photosphere lies within the wind itself. The P Cygni profiles observed in the strong UV resonance lines are formed in this wind. The absorption components of the P Cygni profiles do not start at zero Doppler velocity, because the lower velocity or acceleration layers of the wind are located below the pseudo-photosphere. As the mass loss rate of the wind decreases, the pseudophotosphere moves inwards, and the deeper higher density wind layers become visible, where emission components are formed by recombination and/or collisional excitation. The receding pseudo-photosphere also results in a decrease of the effective radius, which causes an increase of the effective temperature. This explains the increasing degree of ionization, reflected in the observed strengthening of the higher excitation emission lines at the same time that the lower excitation lines become fainter. As the mass loss rate and the wind density decrease further, the emission comes from deeper layers in the wind, where the velocity is smaller, which results in the observed narrowing of the emission lines (see Williams 1992).

(2) Superimposed on the emission lines, one observes two absorption line systems, which are formed in two different shells. The main shell is ejected first and the second shell is ejected later, around day 3 to 5 (see spectra of Andrillat \& Houziaux 1993). The acceleration of the two shells is remarkably similar, which suggests a common mechanism. We propose that the shells are accelerated by radiation pressure in spectral lines (similar to the winds of hot stars) due to the increasing UV brightness of the star.

(3) Within each of the two shells the higher ionization lines have a slightly higher outflow velocity than lower ionization lines, which indicates that the velocity of the shells decreases slightly outwards. This is not due to the gravity of the star, nor to the interaction with material outside the shells, but most likely due to the fact that the velocity slightly increased with time during the ejection of the shells.

(4) The inner shell (second shell), which has a higher velocity than the outer shell (main shell) catches up with the outer shell at about day 50 to 70 . This agrees roughly with the interaction time expected, based on the difference in velocity of the two shells (Sect. 5.2). Also, it explains the rather drastic disappearance of the faster shell system around that date, as well as the sudden increase in velocity of the main shell, and the appearance of hard X-ray flux. From the velocities of the faster secondary shell, of the slower main shell, and the final velocity of the merged shell we estimate that the mass ratio of the main shell to the second shell is roughly $4 / 1$ (Sect. 5.2).

(5) In Sect. 5 we estimated the distance to V1974 Cyg by assuming a spherically symmetric expansion for the nova envelope at the time of the $\mathrm{H} \alpha$ interferometric measurements of Quirrenbach et al. (1993) on day 10, and by combining these with the estimated radius of the principal system at 
the same date. The value so obtained, $2.9 \mathrm{kpc}$, is in good agreement with the independent estimates by Quirrenbach et al. (1993), Della Valle \& Livio (1995) and Paresce et al. (1995).

Our model is qualitatively in agreement with the optically thick wind models of novae, by Kato \& Hachisu (1994), first introduced by Friedjung (1966a,b,c), and further treated by Kovetz (1998) and Bath \& Harkness (1989), who showed that the decreasing mass loss rate of the wind will result in an inward moving photosphere, an increase in the effective temperature and an increase in the degree of ionization in the wind. Since in these models matter is accelerated deep inside the pseudophotosphere, it is possible in this way to explain the formation of the peculiar P Cygni profiles seen in the earliest spectra and the progressive narrowing of the emission lines. Also, because of the predicted inward recession of the pseudo-photosphere and the consequent increase of the effective temperature, it is possible to explain with an unique mechanism the acceleration of the two outward moving shells by radiation pressure in the UV lines. The stratified structure within the expanding shells as suggested in the present model, is in agreement with the major observational features reported by Friedjung $(1966 b, c)$, i.e. the overall decrease of the expansion velocity and of the (Zanstra) temperature with increasing radial distance.

However, our observations and our empirical model require that the nova ejected two shells: one before the discovery at about day -3 , and one at about day 3 to 5 . In this way it is possible to explain why the absorption of the principal system lines is superimposed on the P Cygni profiles of the wind observed on day 1.3 and why the principal and diffuse-enhanced system lines are superimposed on the wind emission lines after day 6 . The first shell contains most of the ejected matter and the second shell, ejected with a higher velocity, contains considerably less material (about $1 / 4$ in this case). We might suggest that the second ejection is the reaction to the main ejection. After the ejection of the main shell, the envelope is out of balance and will try to restore equilibrium, but if this does not succeed immediately, due to some sort of overshooting, a second, less massive shell might be ejected. We like to point out that a similar effect occurs in the large eruptions of luminous blue variables (LBV), which also show that the main eruption is followed by a second one (Humphreys et al. 1999). In the case of LBVs, which are very extended and have a small surface gravity, the main eruption lasts for tens of years, and the second eruption occurs about 60 years after the main one. In the case of eruptions from the surface of white dwarfs, where the gravity is very high, the time scales will be much shorter. It might be interesting to see if dynamical models of nova eruptions show this effect.

According to Friedjung (1987), the principal system shell (here the main shell) is formed by interaction of a wind continuously ejected at a decreasing rate, with material ejected before optical maximum. This model does not explain, however, the presence of the second absorption system with higher velocity, produced inside the main shell. Although Friedjung's interaction model is attractive because wind-shell interaction is likely to occur (see also McLaughlin 1960;
Friedjung \& Duerbeck 1993), it is not clear how this could lead to two shells with strongly different and well separated velocities. On the other hand, as discussed in Sect. 5.2, it is unlikely that the two absorption systems are due to flows in two directions, e.g. one in the orbital plane of the binary and one in the polar direction.

To conclude, we would like to encourage further studies of radial velocity variations in novae (poorly pursued nowdays), especially during the first few days, since they represent a powerful means to get insight into the dynamical structure of the nova envelopes and into the origin of the X-ray emission. Such studies should also provide valuable input information for non-LTE synthetic spectra of novae (see e.g. Short et al. 2001).

Acknowledgements. We are very grateful to Dr. Roberto Viotti for his numerous and helpful comments and to Dr. Michael Friedjung for stimulating this investigation and for constructive criticism. Finally, we are grateful to the anonymous referee for several useful comments and suggestions.

\section{References}

Annuk, K., Kolka, I., \& Leedjärv, L. 1993, A\&A, 269, L5

Andrillat, Y., \& Houziaux, L. 1993, MNRAS, 261, L1

Balman, S., Krautter, J., \& Ögelman, H. 1998, ApJ, 499, 395

Bath, G. T., \& Harkness, R. P. 1989, in Classical Novae, ed. M. F. Bode, \& A. Evans (John Wiley \& Sons Ltd)

Bjorkman, K. S., Johansen, K. A., Nordsiec, K. H., Gallagher, J. S., \& Barger, A. J. 1994, ApJ, 425, 247

Cassatella, A., Benvenuti, P., Heck, A., et al. 1979, A\&A, 74, L18

Cassatella, A., Altamore, A., \& González-Riestra, R. 2002, A\&A, 384, 1023 (Paper I)

Castor, J. I., \& Lamers, H. J. G. L. M. 1979, ApJ, 39, 481

Cecchini, C., \& Gratton, L. 1942, Le Stelle Nuove, ed. U. Hoepli, Milano

Chochol, D., Hric, L., Urban, Z., Grygar, G., \& Papousek, J. 1993, A\&A, 277, 103

Chochol, D., Grygar, J., Pribulla, T., et al. 1997, A\&A, 318, 908

Collins, P. 1992, IAUC, No. 5454

Della Valle, M., \& Livio, M. 1995, ApJ, 452, 704

Friedjung, M. 1966a, MNRAS, 132, 143

Friedjung, M. 1966b, MNRAS, 132, 317

Friedjung, M. 1966c, MNRAS, 133, 401

Friedjung, M. 1987, A\&A, 180, 159

Friedjung, M., \& Duerbeck, H. 1993, in Cataclysmic Variables and Related Objects, ed. M. Hack, \& C. la Dous, NASA SP-507, 371

González-Riestra, R., \& Krautter, J. 1998, ESA SP-413, 367

González-Riestra, R., Cassatella, A., Solano, E., Altamore, A., \& Wamsteker, W. 2000, A\&AS, 141, 343

González-Riestra, R., Cassatella, A., \& Wamsteker, W. 2001, A\&A, 373,730

Humphreys, R. M., Davidson, K., \& Smith, N. 1999, PASP, 763, 1124

Kato, M., \& Hachisu, I. 1994, ApJ, 437, 802

Kovetz, A. 1998, ApJ, 495, 401

Krautter, J., Ögelman, H., Starrfield, S., Wichmann, R., \& Pfeffermann, E. 1996, ApJ, 456, 788

Lamers, H. J. G. L. M., \& Cassinelli, J. P. 1999, Introduction to Stellar Winds (Cambridge Univ. Press) 
Lamers, H. J. G. L. M, Cerruti-Sola, M., \& Perinotto, M. 1987, ApJ, 314,726

Lloyd, H. M., Bode, M. F., O’Brien, T. J., \& Kahn, F. D. 1993, MNRAS, 265, 457

McLaughlin, D. B. 1960, in Stars and Stellar Systems VI, ed. J. L. Greenstein, Stellar Atmospheres, (Chicago: Univ. Chicago Press), 585

Nussbaumer, H., \& Stencel, R. E. 1987, in Exploring the Universe with the IUE Satellite, ed. Y. Kondo et al. (D. Reidel), 203

Paresce, F., Livio, M., Hack, W., \& Korista, K. 1995, A\&A, 299, 823

Quirrenbach, A., Elias II, M. N., Mozurkewich, D., et al. 1993, AJ, 106,1118

Rosino, L., Iijima, T., Rafanelli, P., et al. 1996, A\&A, 315, 463
Saraph, H. E., \& Seaton, M. J. 1980, MNRAS, 193, 617

Shore, S. N., Sonneborn, G., Starrfield, S., González-Riestra, R., \& Ake, T. B. 1993, AJ, 106, 2408

Shore, S. N., Sonneborn, G., Starrfield, S., González-Riestra, R., \& Polidan, R. S. 1994, ApJ, 421, 344

Shore, S. N., Starrfield, S., Ake III, T. B., \& Hauschildt, P. H. 1997, ApJ, 490, 393

Short, C. I., Hauschildt, P. H., Starrfield, S., \& Baron, E. 2001, ApJ, 547,2001

Slavin, A. J., O’Brien, T. J., \& Dunlop, J. S. 1995, MNRAS, 276, 353

Williams, R. E. 1992, AJ, 104, 725

Woodward, C. E., Greenhouse, M. A., Gehrz, R. D., et al. 1995, ApJ, 438,921 